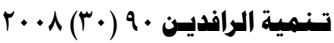 \\ ص ص
}

\section{العوامل الرئيسة المؤثرة على الأهمية النسبية للناتج الزراعي \\ في دول نامية مختارة}

الدكتورة آلاء محمد عبد الله

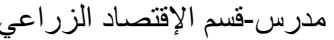

كلية الزر اعة و الغابات-جامعة الموصئل الزيل

aaalasam@yahoo.com

\section{|المستخاصن}

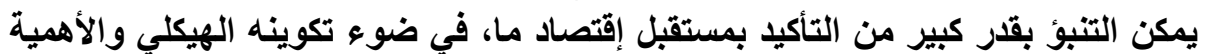

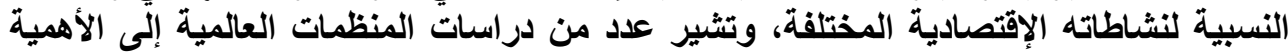

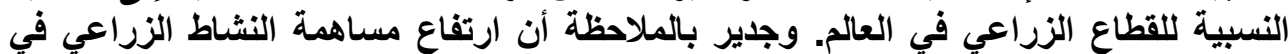

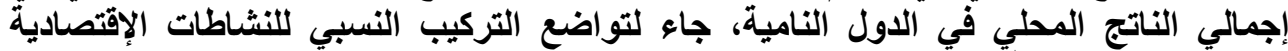

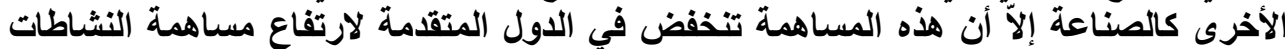

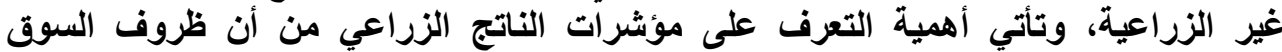

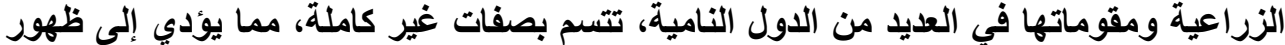

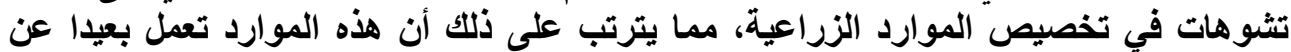

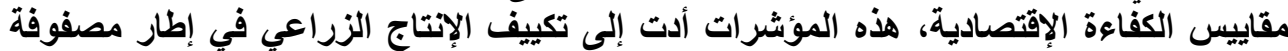

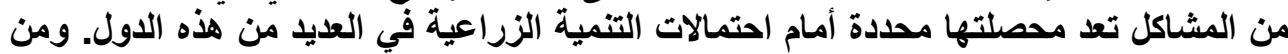

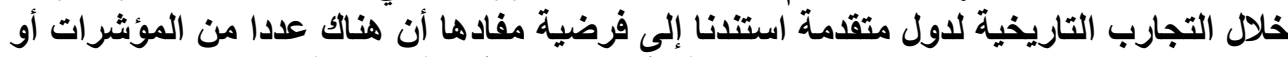

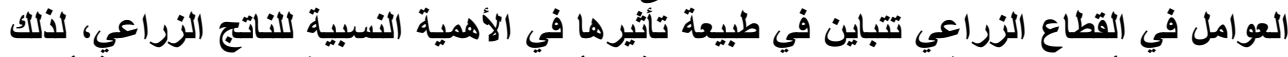

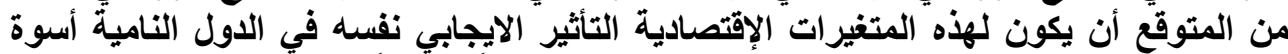

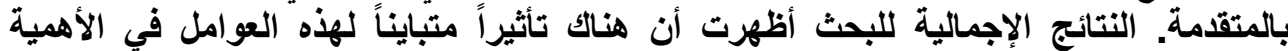

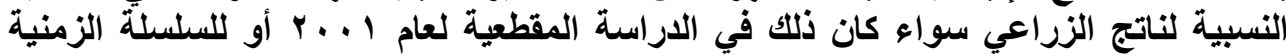

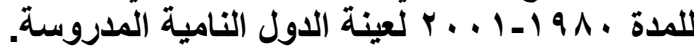




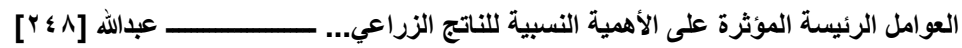

\title{
The Basic Factors Affecting the Relative Importance of Agricultural Product in a Selected Developing Countries
}

\author{
Alaa M. Abdullah (PhD) \\ Lecturer \\ Department of Agricultural Economy \\ University of Mosul
}

\begin{abstract}
It is possible however to forecast much greatly in future of certain economy in the light of structural formulation, relative importance and other various economic activities. The international organizations referred to the relative importance of the agricultural sector in the world. It is worth mentioning that the high participation of agricultural activity in GDP in developing countries has come to be with the relative structure of other economic activities such as industry. But this participation may be reduced in advanced counties due to the rise of participation of activities other than agricultural. This importance comes to consider the conditions of agricultural markets in so many developing countries. This will lead to the emergence of unbalances in allocating agricultural resources. These resources may work far from the standards or measurements of economic efficiency. These indicators have led to the adjustment of agricultural production in the term of a matrix of problems and the result is limited in front of agricultural development and its probability in many of these countries. Through historical experiments of advanced states, we have relied upon a hypothesis which tells that there are a number of indicators inside agricultural sector vary with respect to the nature of its effect upon relative importance of the agricultural product .Therefore, it is expected that these economic variances have appositive effect the same in developing states.
\end{abstract}

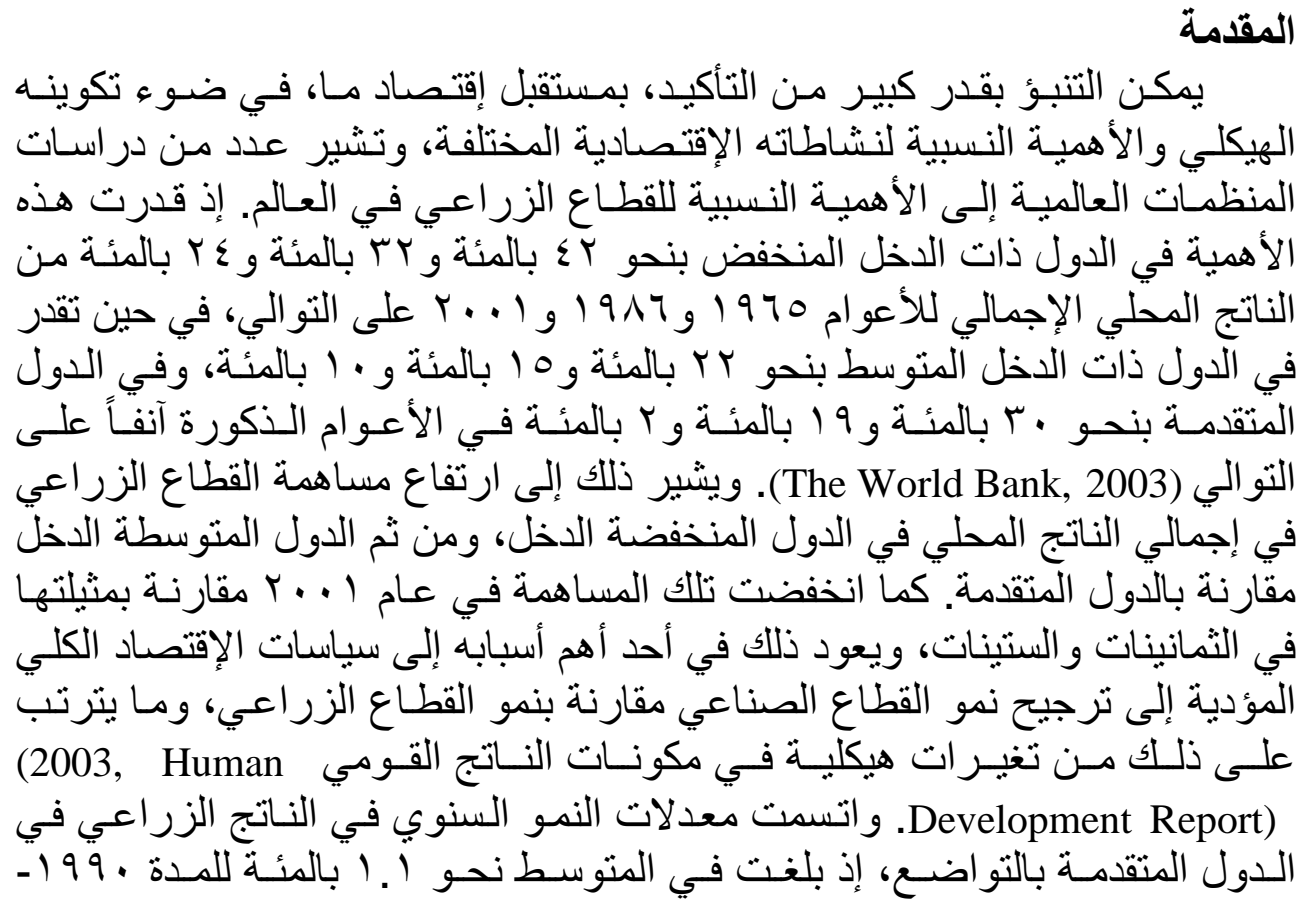




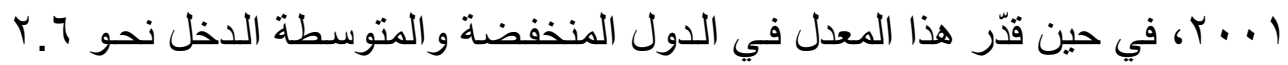

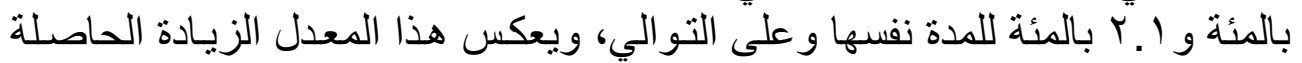

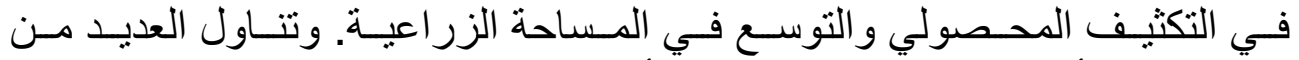

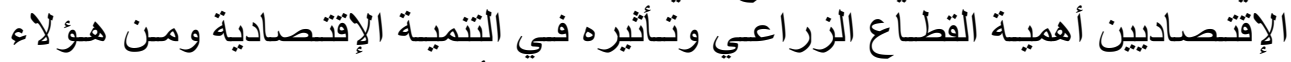

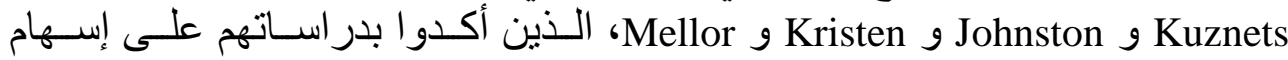

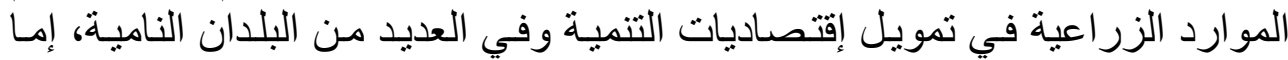

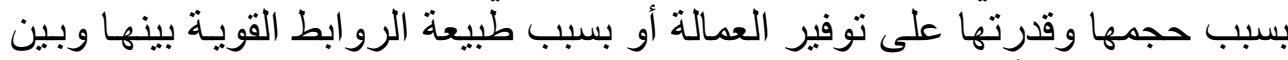

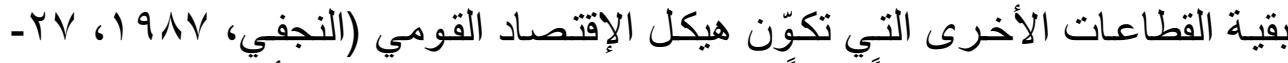

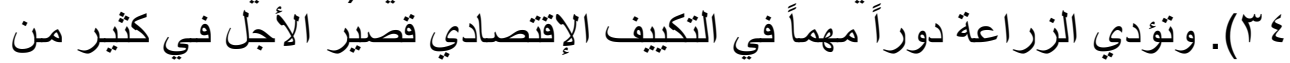

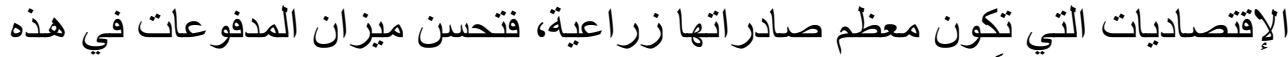

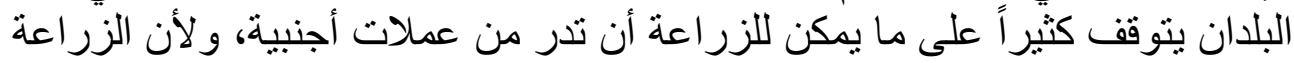

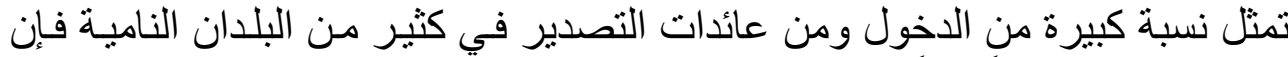

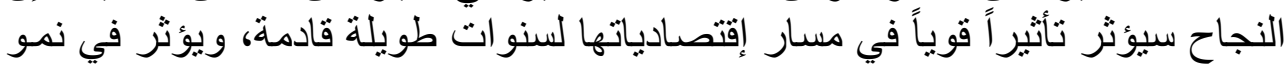

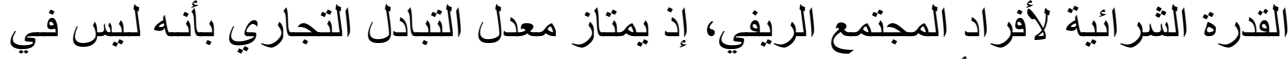

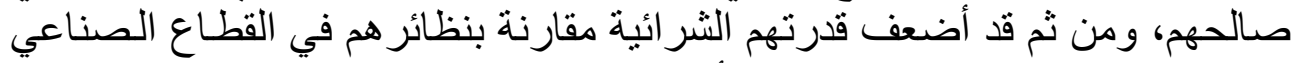

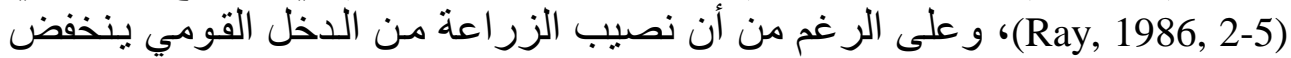

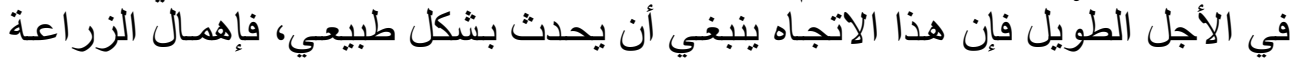

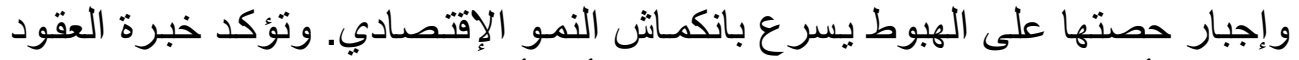

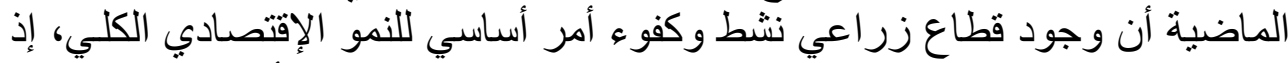

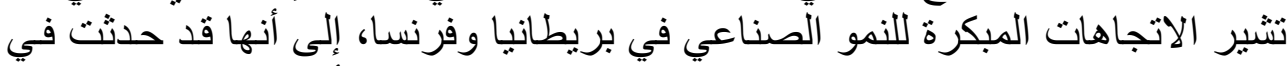

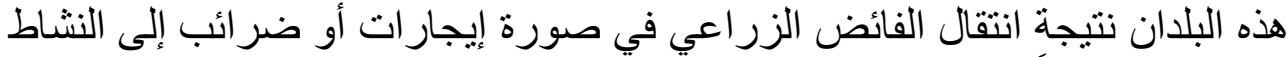

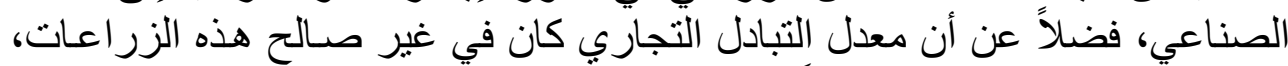

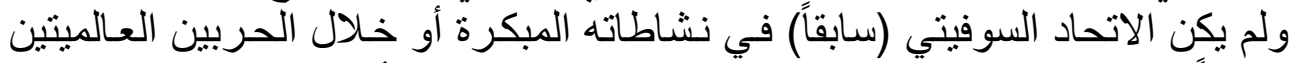

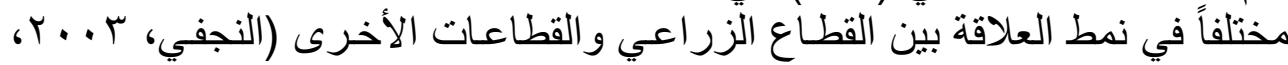
.(^9-^r

الأهمية

تتأثز مؤشرات الأداء للجهاز الإنتاجي للقطاع الزراعي إلى درجة كبيرة

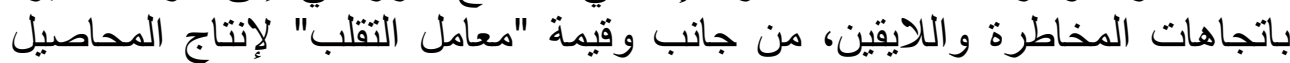

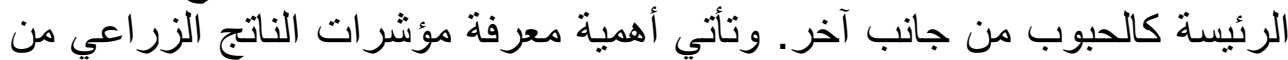

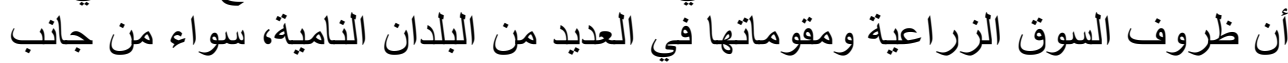

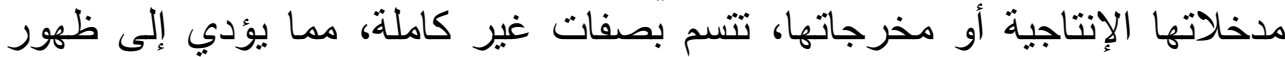

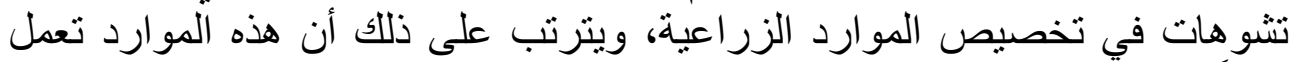

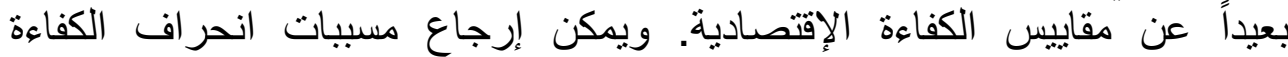

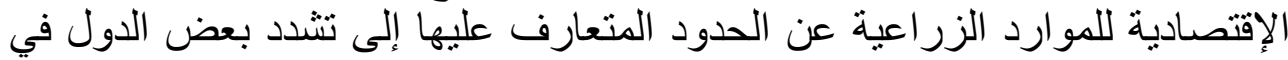

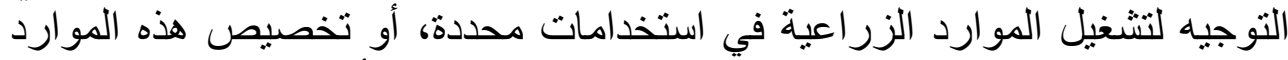
في إنتاج محاصيل زر اعية معينة من دون غير ها في إطار الأمن الغذائي . 
المشكلة

أدت مؤشرات الناتج الزراعي في زراعة البلدان النامية إلى تكييف الإنتاج

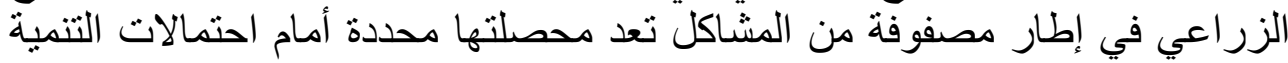
الزر اعية في العديد من هذه الإقتصاديات.

يرتبط نجاح الخطط الإقتصادية الزراعية بعدة عوامل بوصفها من المؤشرات

الهرف

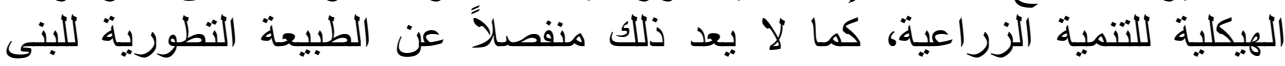

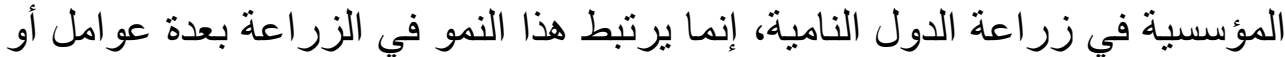

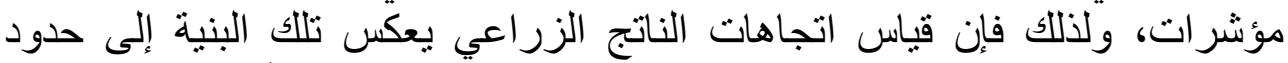

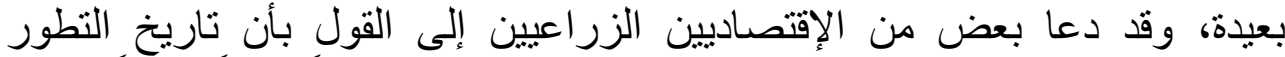

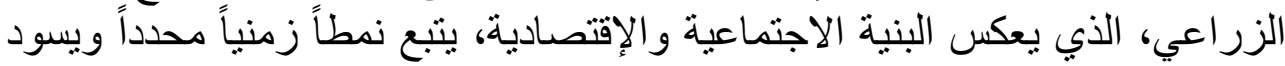
في معظم دول العالم بصرف العكب النظر عن مستوباتها الدخلية.

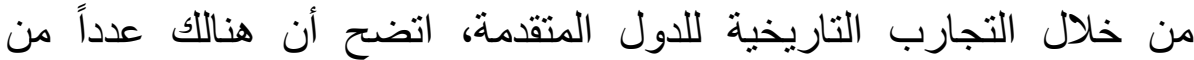

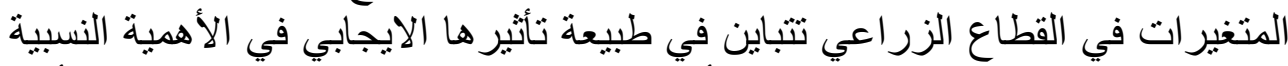
للناتج الزراعي، لذلك من المتوقع أن يكون لهذه المتغيرات الإقتصادية التأثير الايجابي نفسه في الدول النامية أسوة بالمتقدمة.

\section{الأنموذج القياسي المستخدم في التقدير}

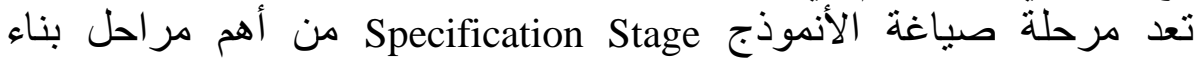

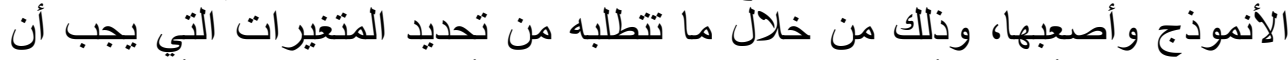

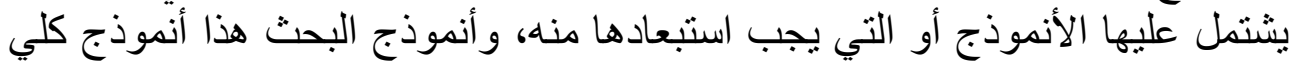

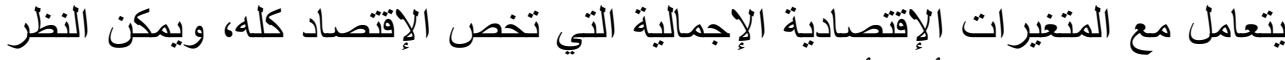

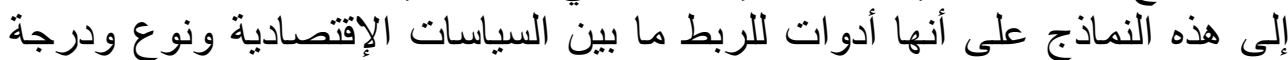

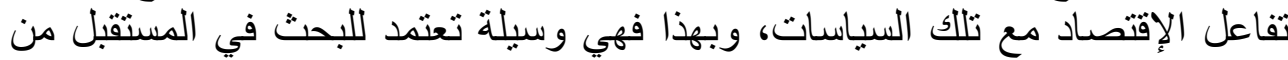

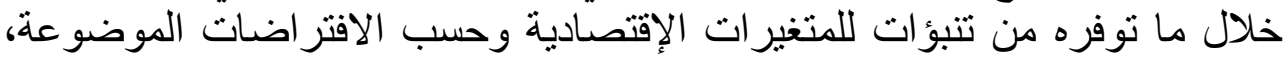

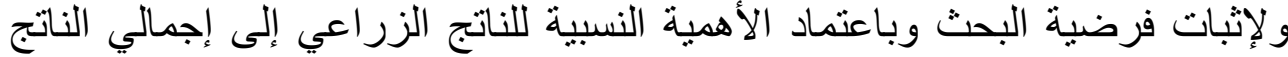

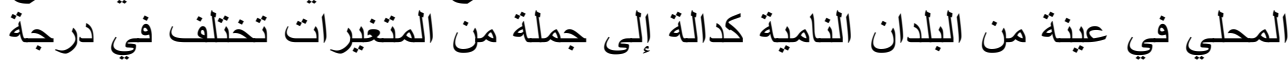

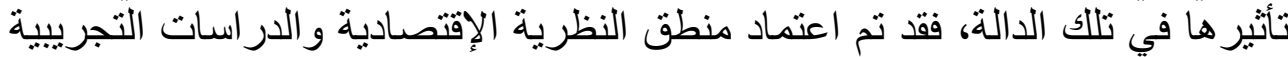

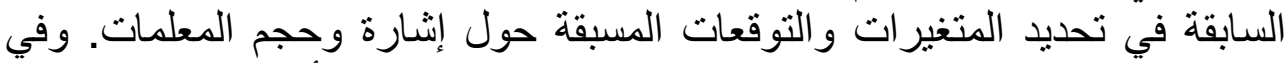

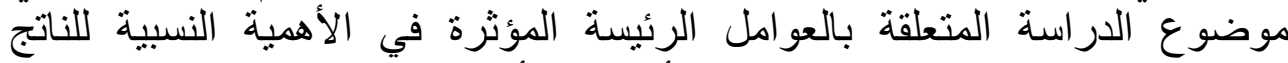

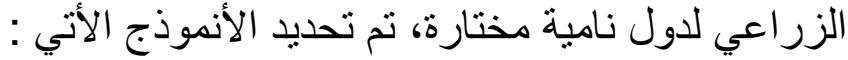

$\mathrm{Y}=\mathrm{B}_{\mathrm{O}}+\mathrm{B}_{1} \mathrm{X}_{1}+\mathrm{B}_{2} \mathrm{X}_{2}+\mathrm{B}_{3} \mathrm{X}_{3}+\mathrm{B}_{4} \mathrm{X}_{4}+\mathrm{B}_{5} \mathrm{X}_{5}+\mathrm{B}_{6} \mathrm{X}_{6}+\mathrm{B}_{7} \mathrm{X}_{7}+\mathrm{Ui}$ 
نسبة الناتج الزر اعي إلى الناتج المحلي الإجمالي (المتغير المعتمد) = Y

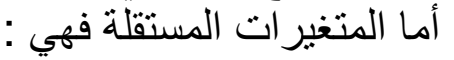

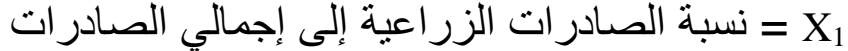

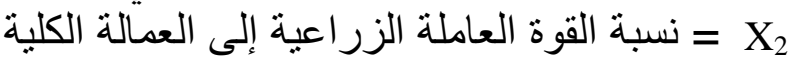

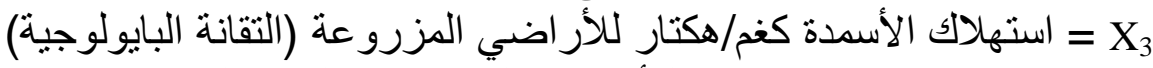

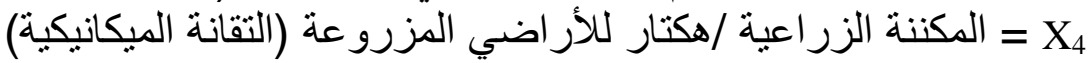
نسبة النفقات الحكومية إلى الناتج المحلي الإجمالي (مقياس لحجم دور الحئ ل

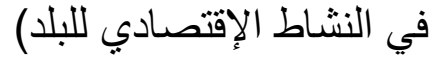
X = المساحات الإنتاجية /كم وتعكس اتجاهات المزارعين بالتوسع في الإنتاج

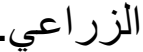

= X7

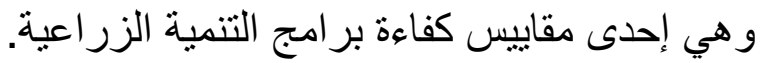

Bo

= معاملات المتغير ات Coefficient Bi = Ui

أما فيما يتعلق بيلّان موضوع البنو البحث فقد تم اختيار 17 بلداً نامياً ذات متوسط

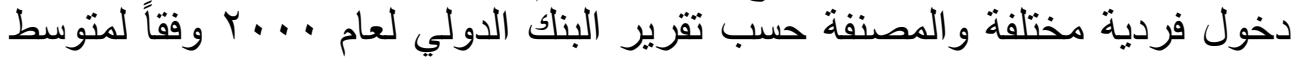
دخل الفرد من الناتج القومي الإجمالي (The World Bank Group, 2002) وتمثلت ودئت

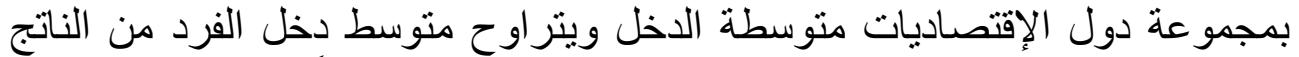

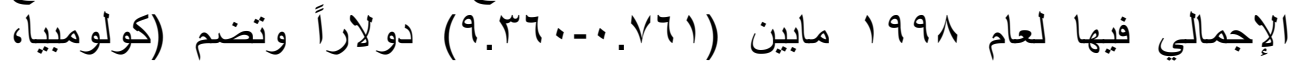
إكوادور، مصر، السلفادور، هنداروس، فلبين، رومانيا، الاتحاد الروسي، تايليندا، الإنا،

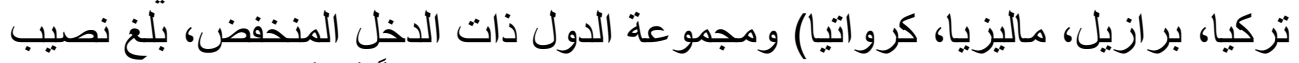

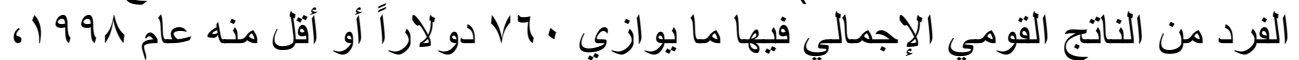

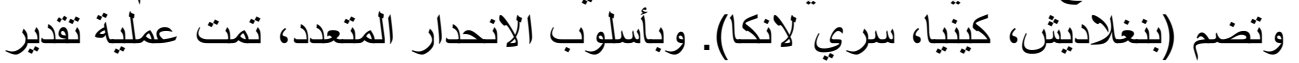

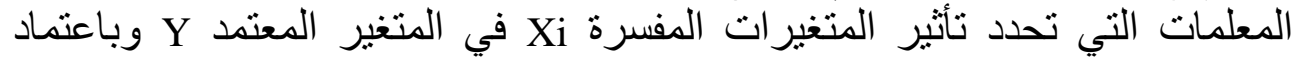

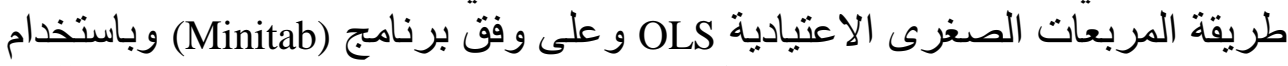

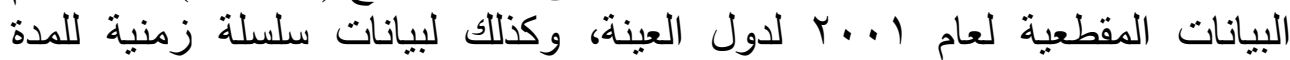

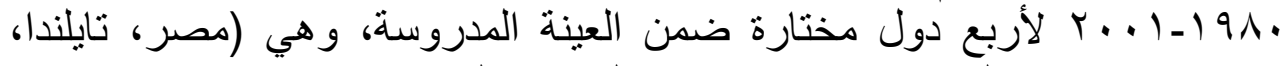

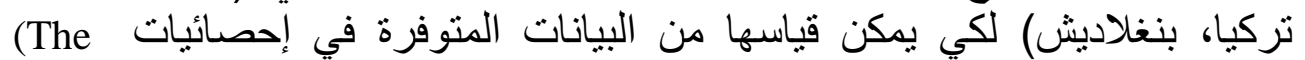
World Bank, 2003)

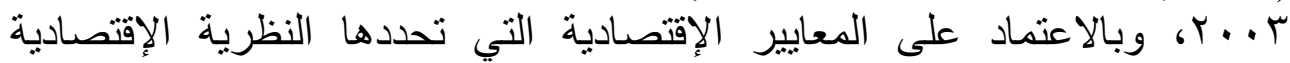

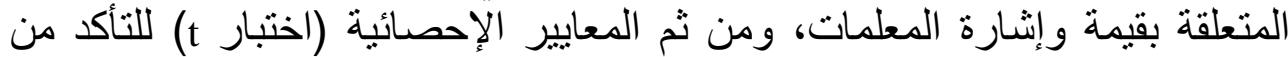

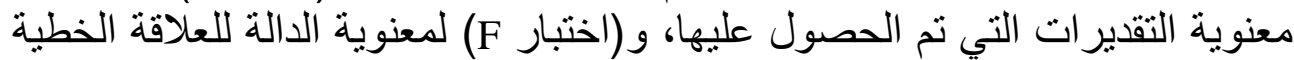

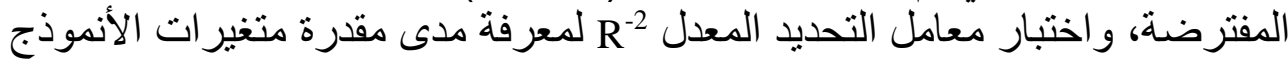

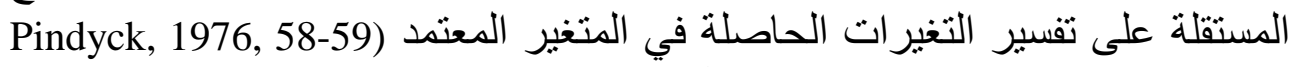
Rubifeld \&

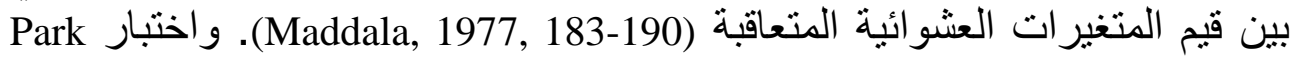




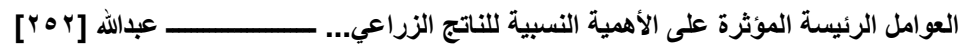

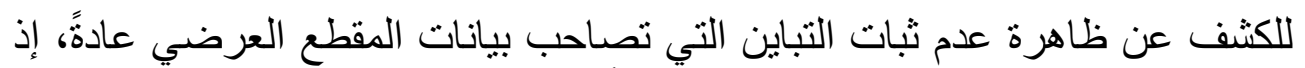

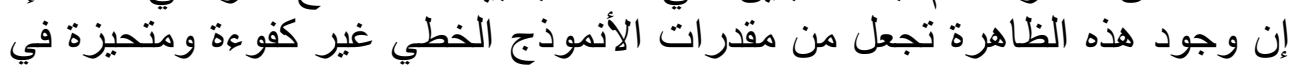

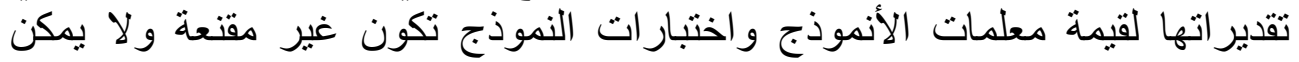

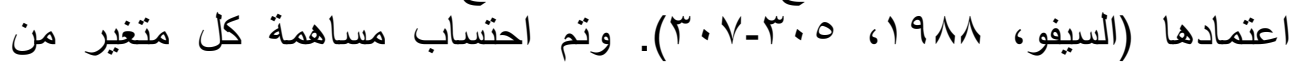

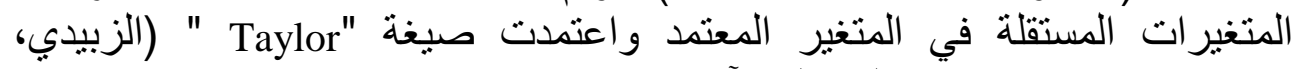

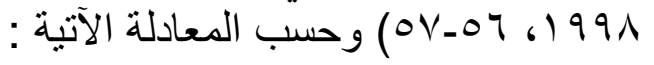
$\mathrm{Y}=\mathrm{b} 1\left(\mathrm{y}^{-} / \mathrm{x}^{-}\right) \Delta \mathrm{x}_{1}+\mathrm{b} 2\left(\mathrm{y}^{-} / \mathrm{x}^{-}\right) \Delta \mathrm{x}_{2}+\mathrm{b} 3\left(\mathrm{y}^{-} / \mathrm{x}^{-}\right) \Delta \mathrm{x} 3+\mathrm{b} 4\left(\mathrm{y}^{-} / \mathrm{x}^{-}\right) \Delta \mathrm{x}_{4}+\mathrm{b} 1\left(\mathrm{y}^{-} / \mathrm{x}^{-}\right)$ $\Delta 5+\mathrm{b} 6\left(\mathrm{y}^{-} / \mathrm{x}^{-}\right) \Delta \mathrm{x}_{6}+\mathrm{b} 7\left(\mathrm{y}^{-} / \mathrm{x}^{-}\right) \Delta \mathrm{x}_{7}$

إذ تمثل :

Y : المتغير المعتمد (نسبة الناتج الزر اعي إلى الناتج المحلي الإجمالي). xi”)

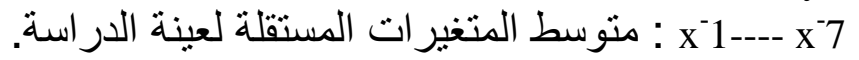

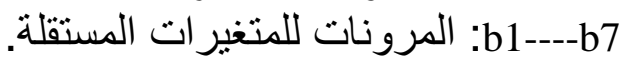

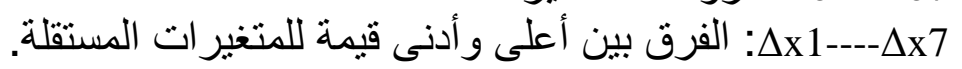

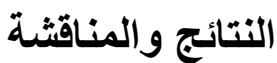

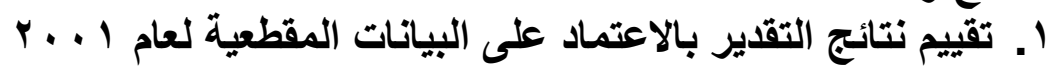

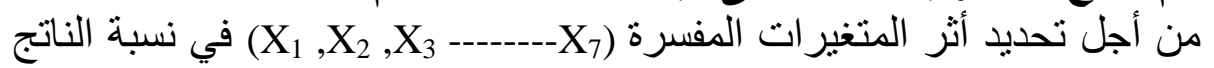

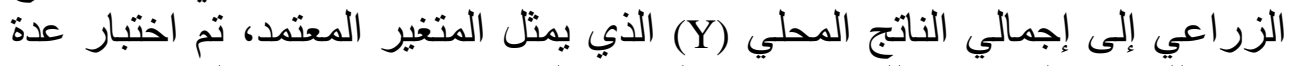

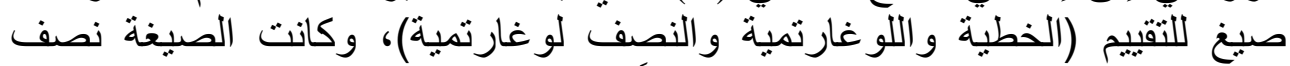

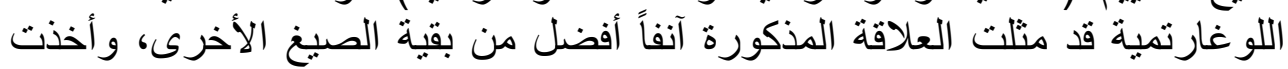

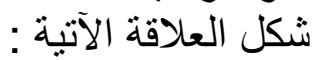

$$
\begin{aligned}
\log \mathrm{Y}= & 1.78+0.066 \mathrm{X}_{1}+0.009 \mathrm{X}_{2}+0.084 \mathrm{X}_{3}-0.001 \mathrm{X}_{4}+0.021 \mathrm{X}_{5} \\
\mathrm{t}^{*}= & (7.36) \quad(2.45) \quad(2.08) \quad(2.35) \\
& -0.113 \mathrm{X}_{7}
\end{aligned}
$$

معادلة | (1) - مع

$$
\mathrm{R}^{-2}=64.5 \%
$$

$$
\mathrm{F}=5.55 \quad \text { D.W }=2.56
$$

من نتائج التقدير الموضحة آنفاً، استطاعت المتغير ات المفسرة بواسطة قيمة

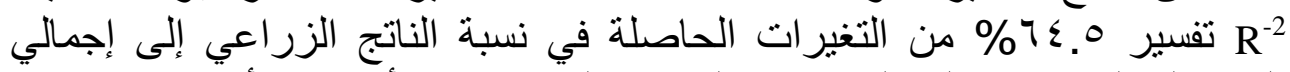

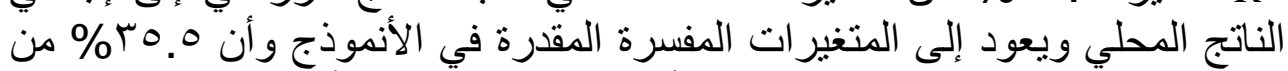

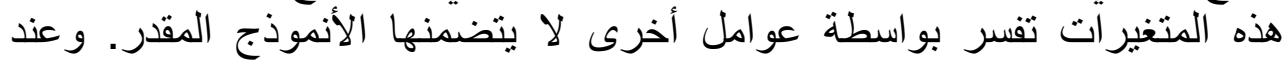

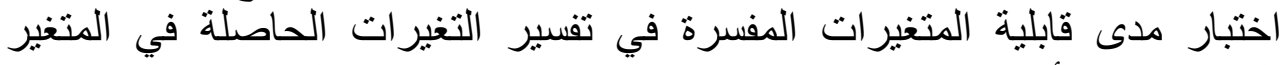

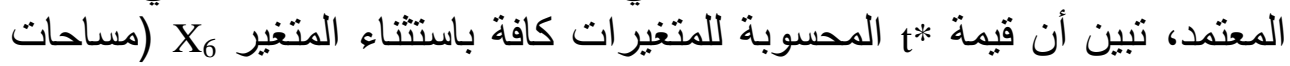

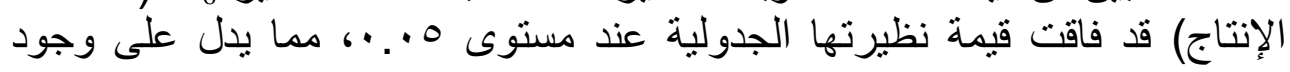




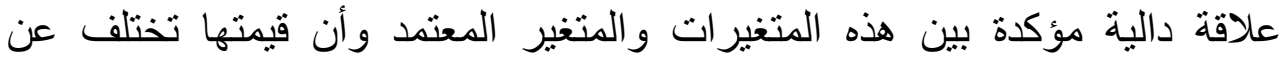

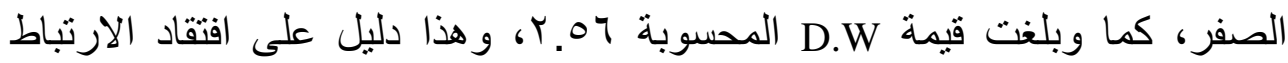

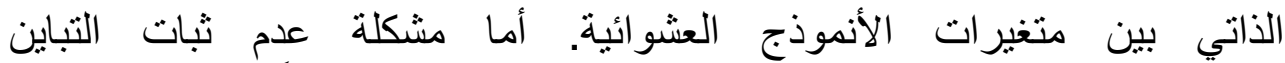

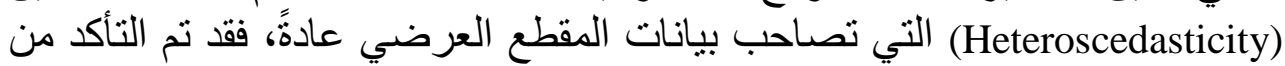
عدم وجودها من اختبار Park، وذللك بإجر اء الانحدار بين لو غاريتم مجموع مربعات التعات

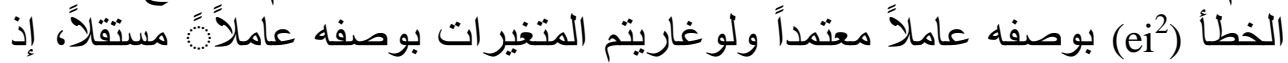

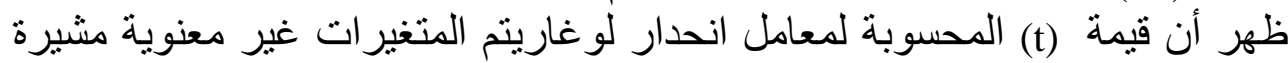
إلى عدم وجود هذه الظاهرة. وأوضحت نتائج التقدير أن الأنموذج الكير الكلي أخذ الصيخة

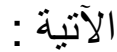

$\log \mathrm{Y}=1.77+0.061 \mathrm{X}_{1}+0.008 \mathrm{X}_{2}+0.076 \mathrm{X}_{3}-0.001 \mathrm{X}_{4}+0.021 \mathrm{X}_{5}$

$$
\begin{aligned}
& \mathrm{t}^{*}=(7.03) \quad \begin{aligned}
&(2.08) \\
&+0.024 \mathrm{X}_{6}--0.114 \mathrm{X}_{7} \\
&(0.49) \quad(-2.50)
\end{aligned} \\
& \mathrm{R}^{-2}=61.3 \% \\
& \mathrm{~F}=4.39 \quad \mathrm{D} . \mathrm{W}=2.47
\end{aligned}
$$

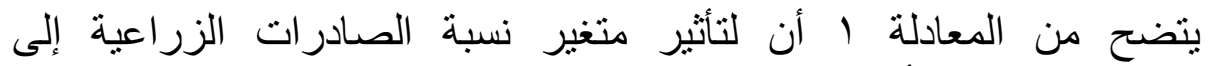

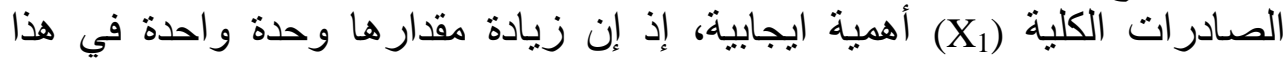

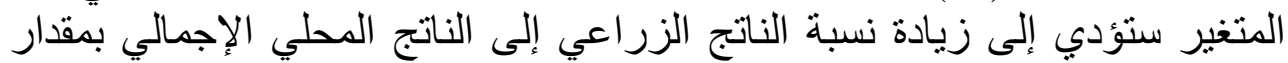

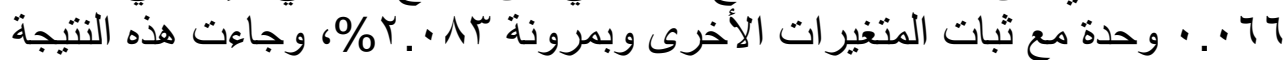

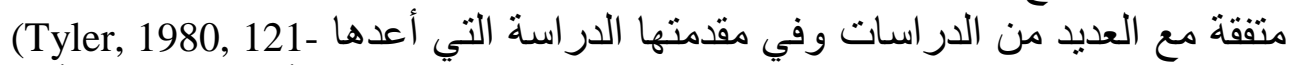

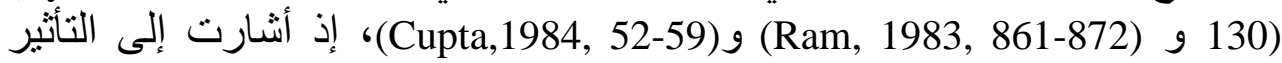

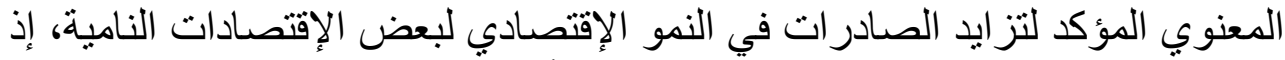

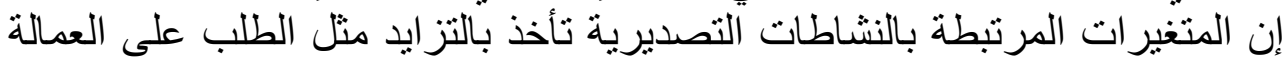

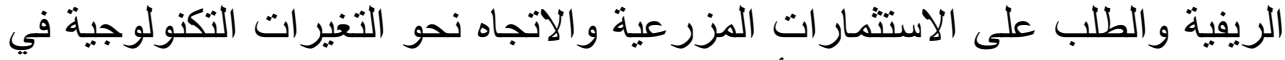

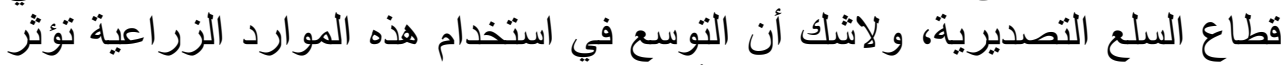

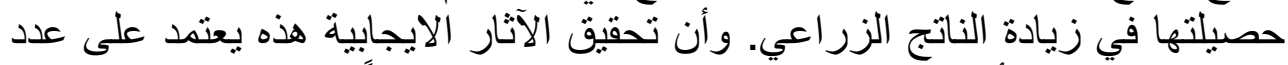

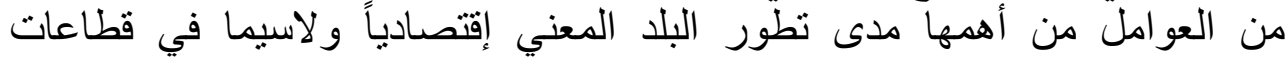
الصناعة ومستوى الإنتاجية فيه ومدى الكفاءة الإقتصادية في قطاعاتهاته الإنتاجية

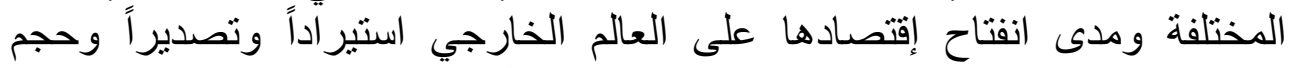

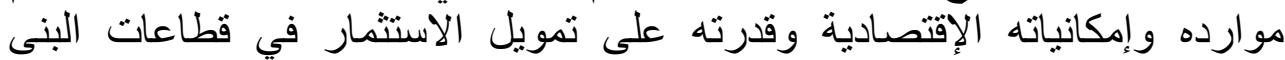

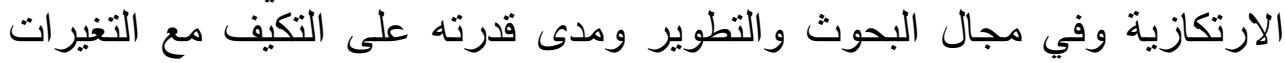

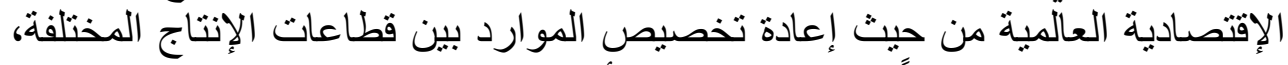

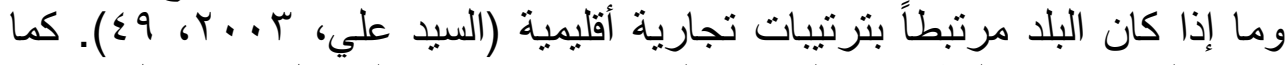

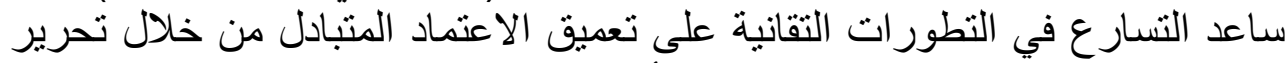

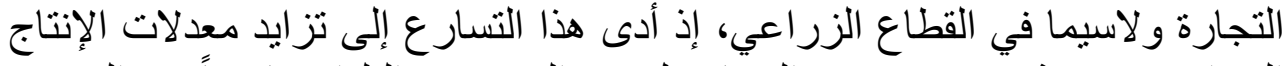

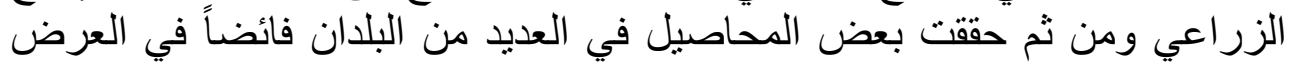




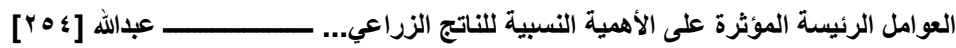

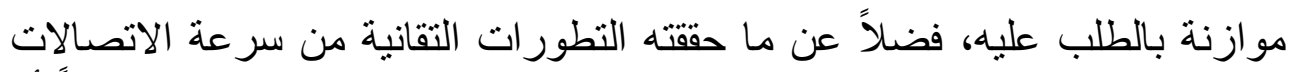

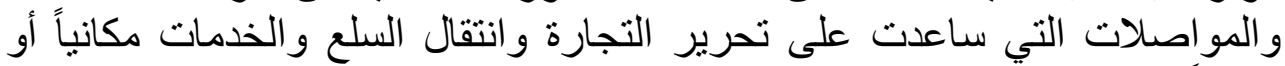

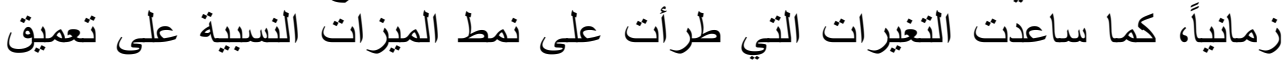

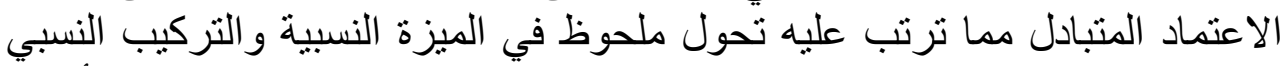

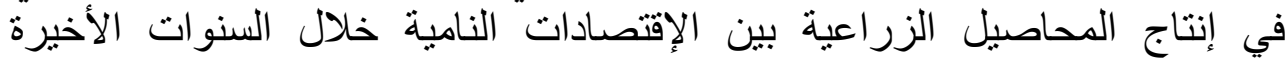

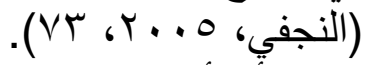

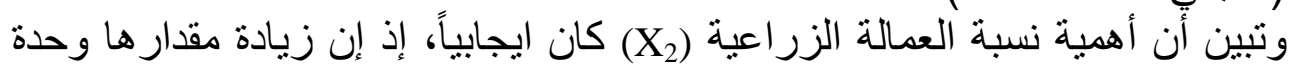

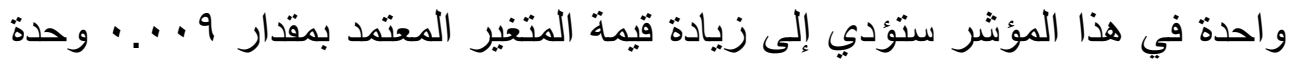

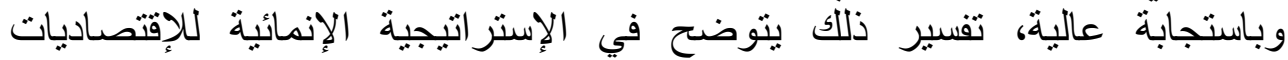

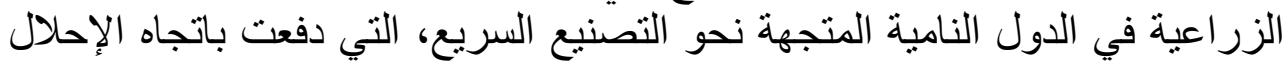

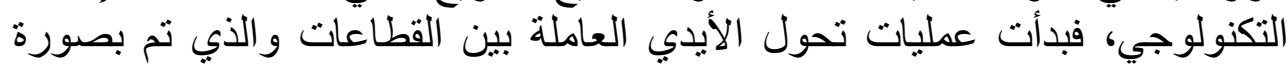

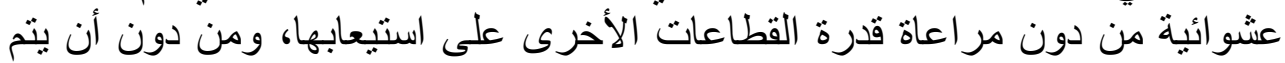

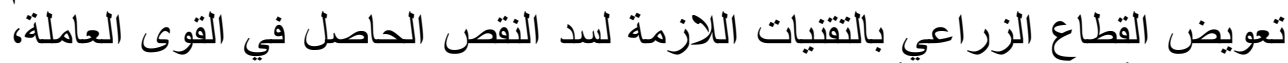

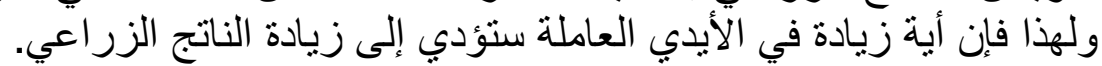

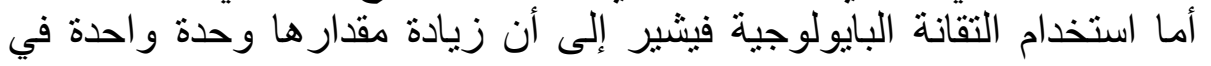

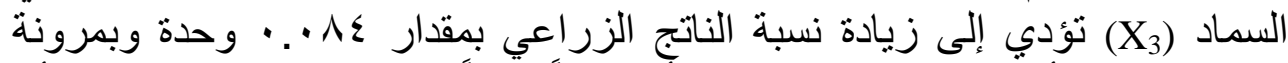

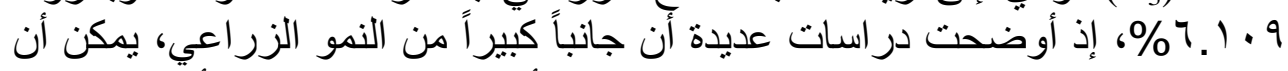

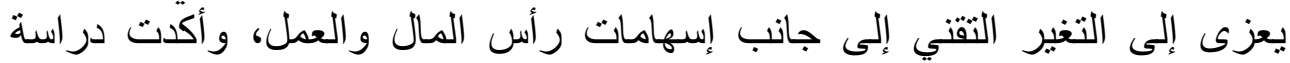
(H.Binswanger, 1989)

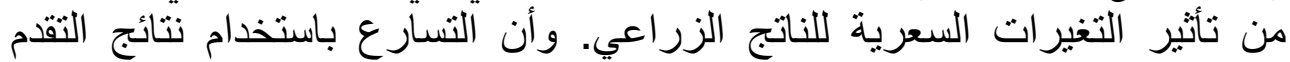

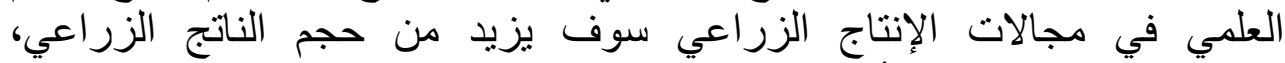

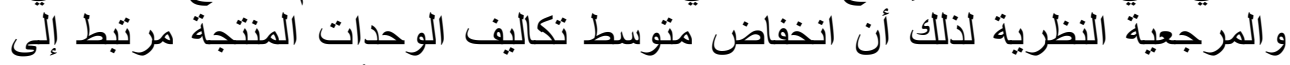

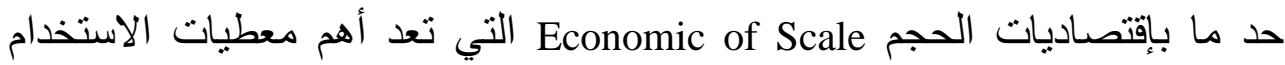

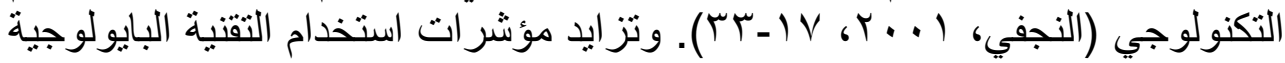

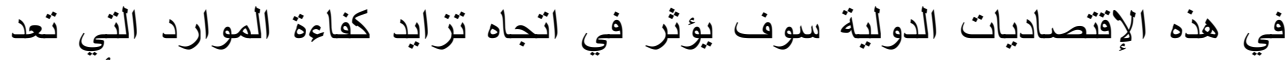

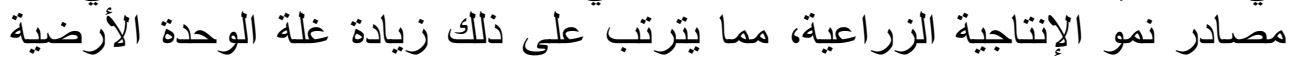

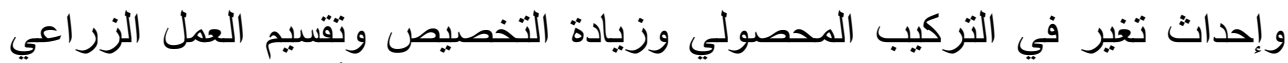
والاتجاه نحو الميزة النسبية في الإنتاج الزراعي التي يمكن أن تؤدي إلى معدلات نمو متسار عة. وفيما بتعلق بالتقانة الميكانيكية (X4) ظهر أن زيادة استخدام هذه التقانة بمقدار

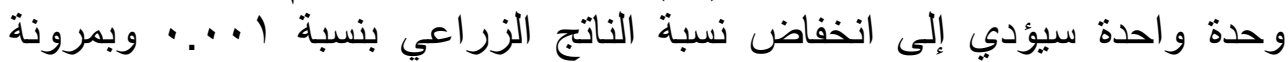

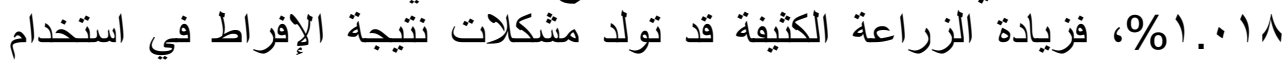

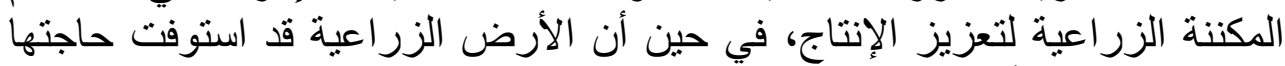

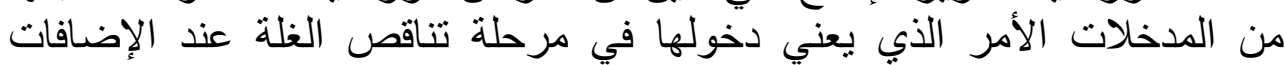

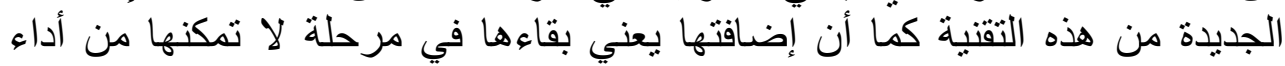

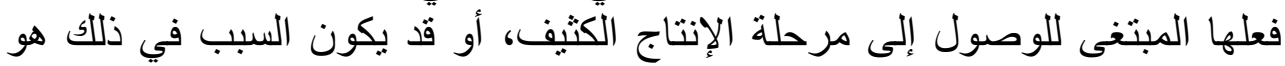

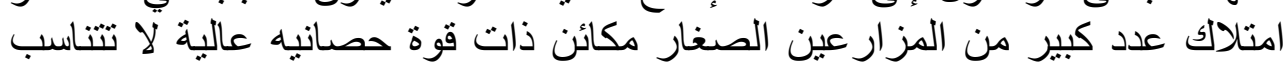


والحيازة المزرعية التي يستغلونها ومن ثم هنالك كم هائل من هذه القوى معطلة

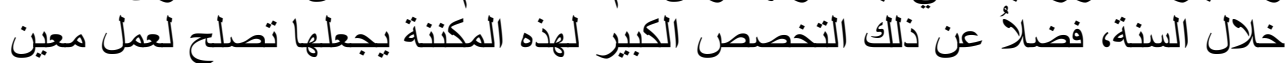

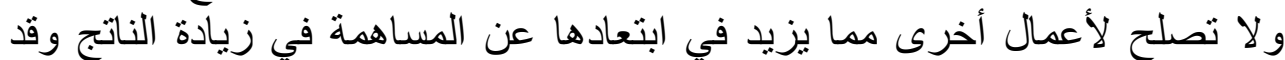

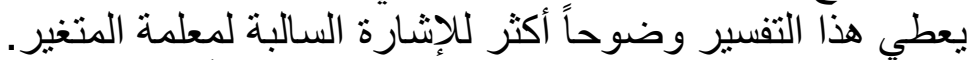

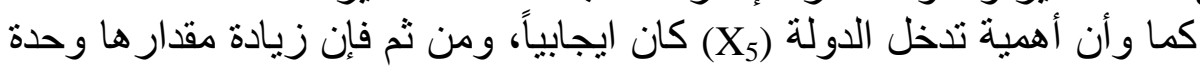

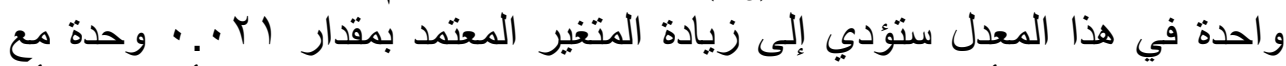

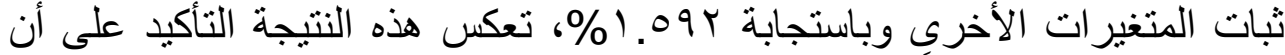

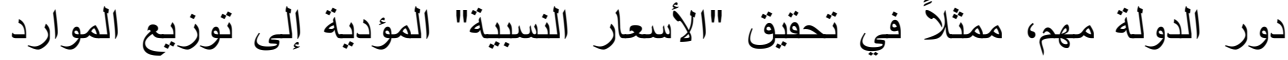

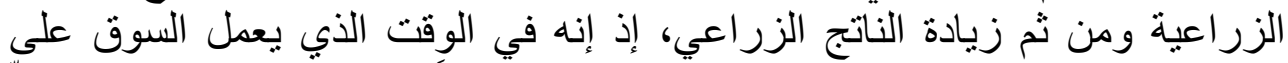

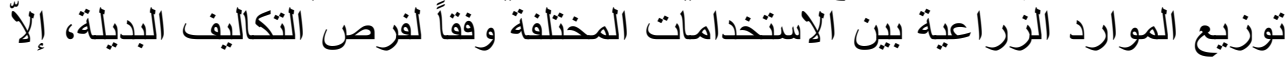

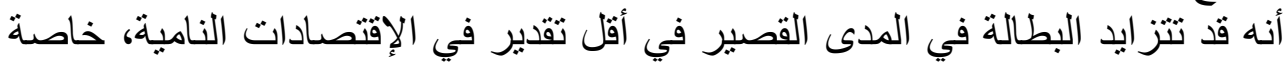

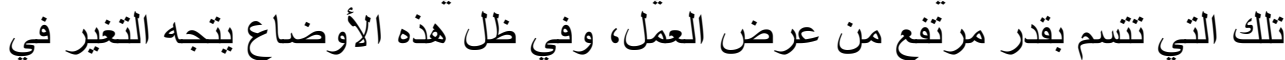

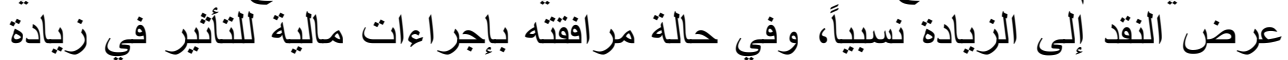

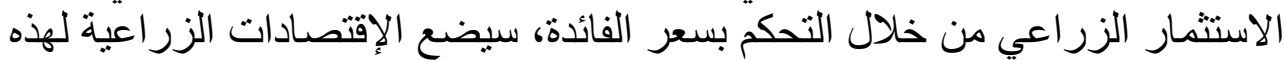

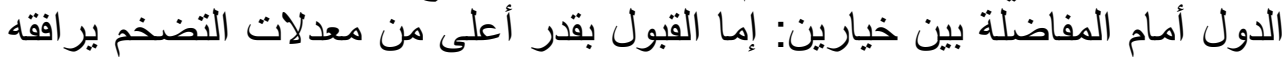

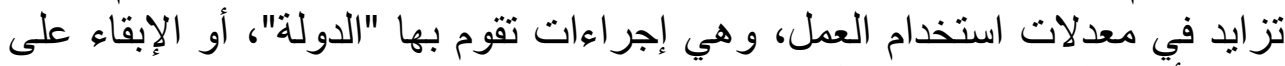

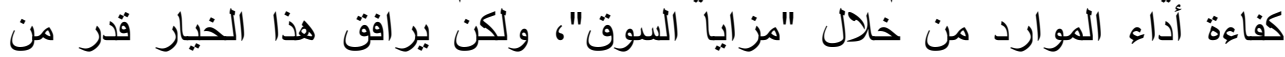

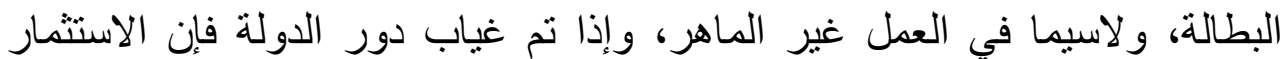

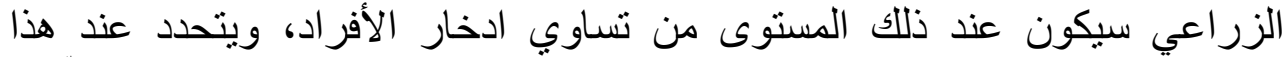

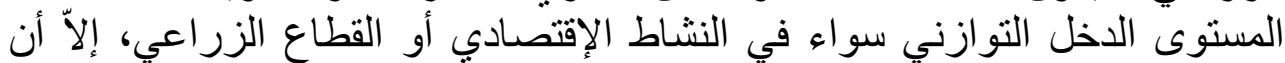

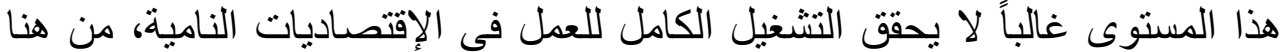
فإن توسيع هذا الدور وتفعيله في تنشيط الاستثمار من خلال الإل إعادة تركيب هيكل

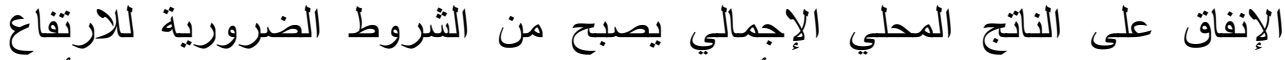

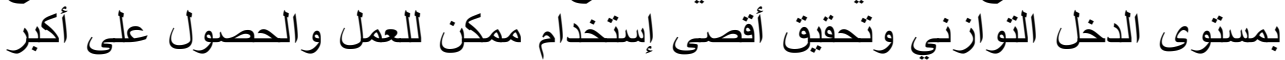

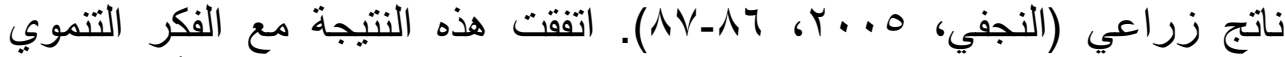

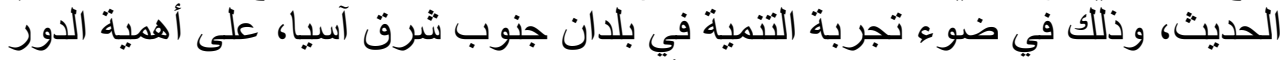

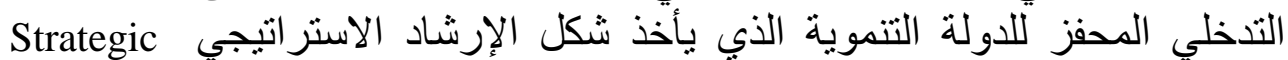

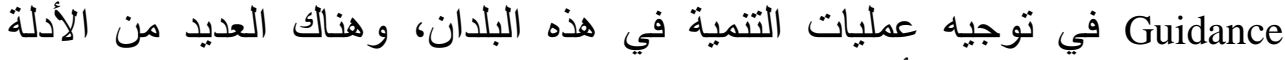

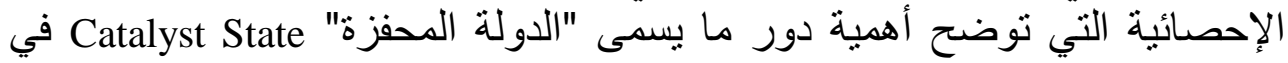
إحداث تغييرات كبيرة وجوهرية في التركيبة الإقطاعية للبلدان حديثة التبنة التية، و هدفت تللك الإستراتيجية إلى تحقيق تحول نوعي في في البنية الإقتصادية والتية التركيبة

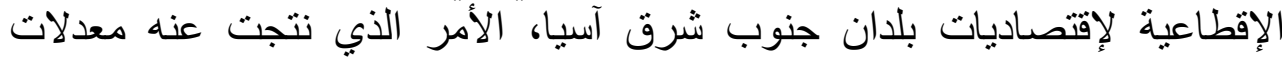

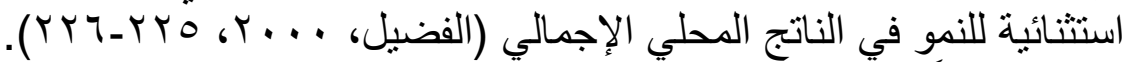

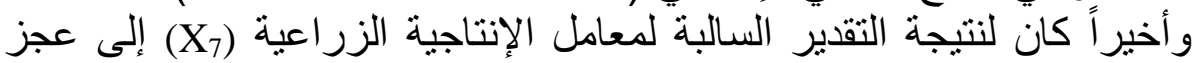

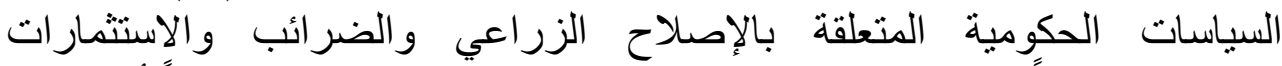

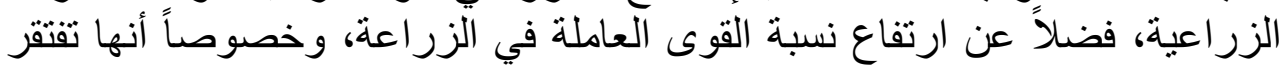

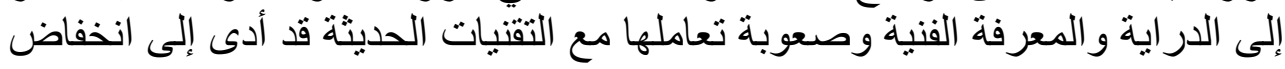




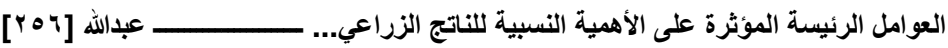

الإنتاجية الزراعية، وهذا مما يؤكد غياب إستراتيجية زراعية على صعيد الدول النامية تسعى إلى تحديد اتجاهات النمات النمو.

r. التقييم الإقتصادي والكمي لنتائج التقدير لاول مختارة من عينة الاراسة

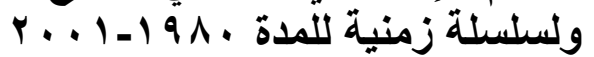

أولاًا - تايلند

تمتاز تايلند بوفرة مواردها الزراعية، فطبيعة الظروف المناخية فئاخ في هذه الدولة

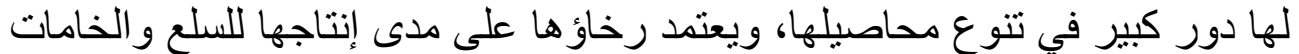

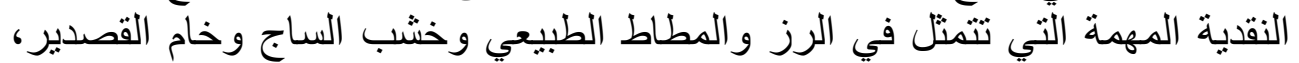

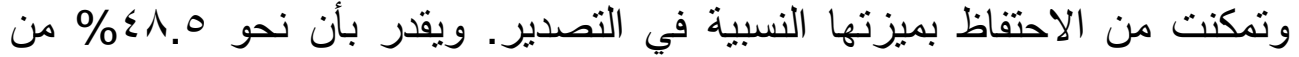

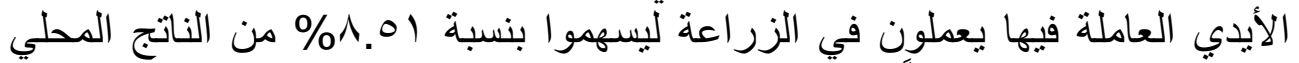

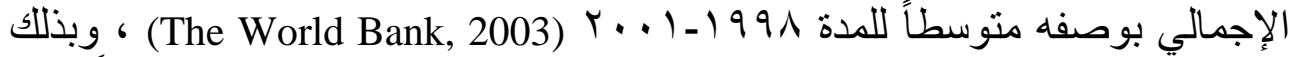

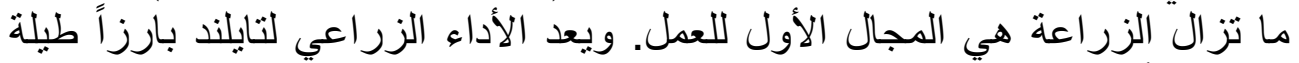

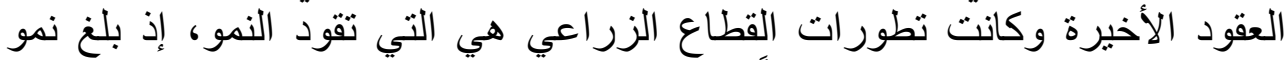

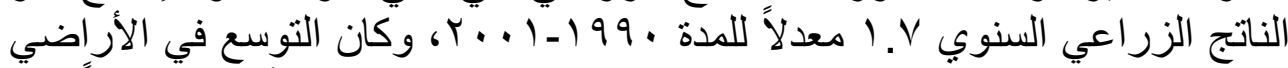

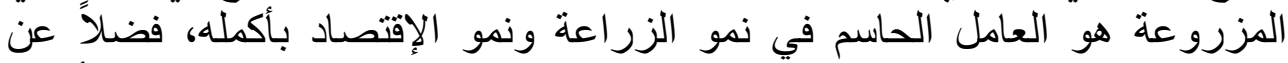

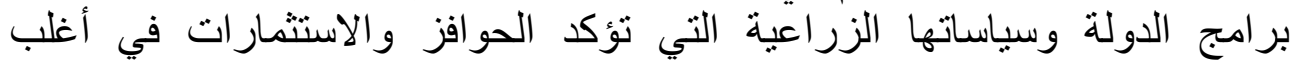

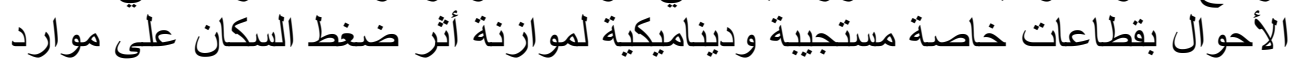
الأرض. كما أن تنويع وتحسين إنتاجية الحاصلات الموجودة من الأمور الحيوية

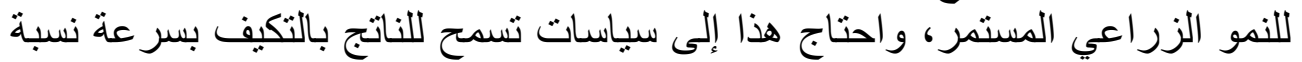

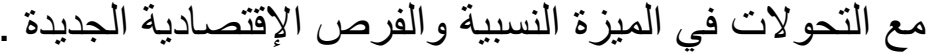
أظهر التحليل الكي أن الدالة الخطية أفضل الدوال الدئلة المقدرة وهي بالثكل الآتي

$\mathrm{Y}=85.9+0.294 \mathrm{X}_{1}-3.52 \mathrm{X}_{2}-0.431 \mathrm{X}_{5}$

المعادلة ب

$\mathrm{R}^{2}=95.0 \%$

$\mathrm{F}=75.5 \quad$ D. $\mathrm{W}=1.88$

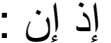

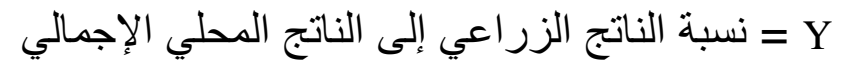

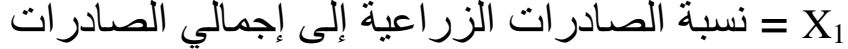

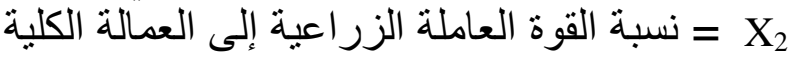

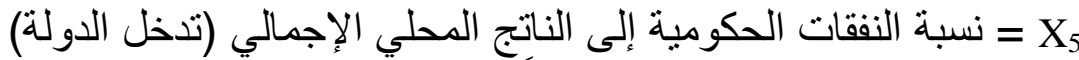

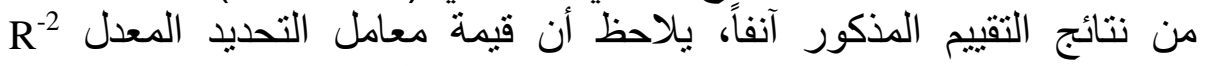

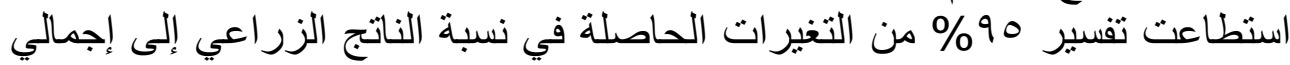

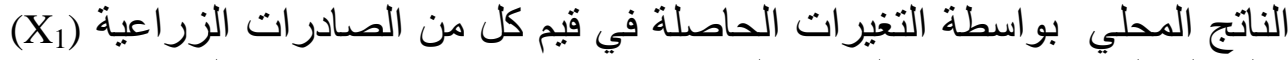

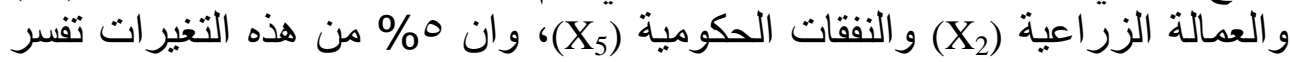

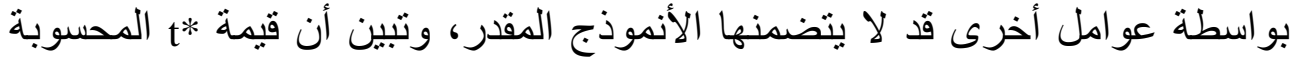

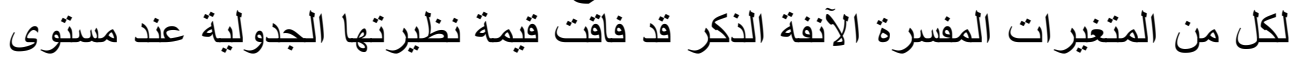


معنوية ه.. •، مما يدل على وجود علاقة دالية مؤكدة بين المتغير المعتمد

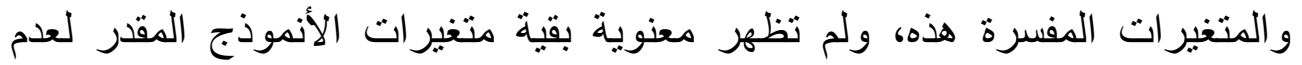
مقدرتها على اجتياز الاختبار ات الإحصائية.

وأوضحت نتائج التقدير أن الأنموذج الأني أخذ الصيغة الآتية :

$\mathrm{Y}=93.3+0.438 \mathrm{X}_{1}-3.77 \mathrm{X}_{2}-0.0008 \mathrm{X}_{3}+0.012 \mathrm{X}_{4}$

$\mathrm{t}^{*}=(6.54) \quad(1.65) \quad(-5.13) \quad(-0.21) \quad(0.89)$

$-0.641 \mathrm{X}_{5}-0.0007 \mathrm{X}_{6}$

$(-2.11) \quad(-0.30)$

$\mathrm{R}^{-2}=99.9 \%$

$\mathrm{F}=66.57$

D. $\mathrm{W}=1.67$

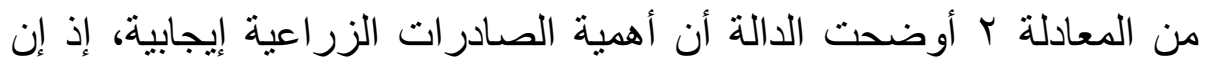

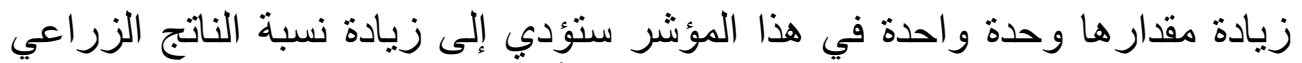

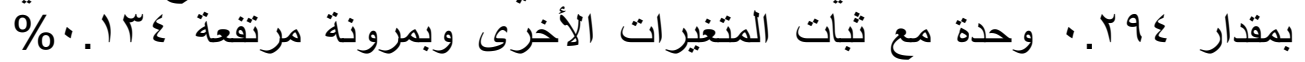

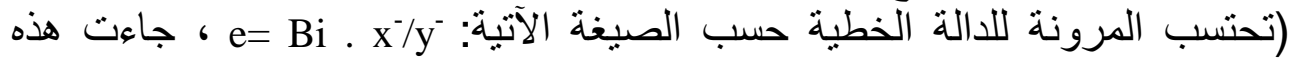

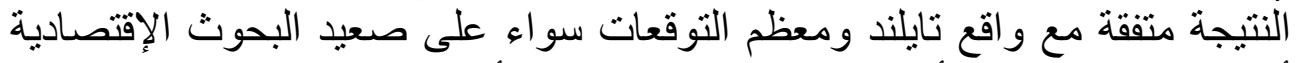

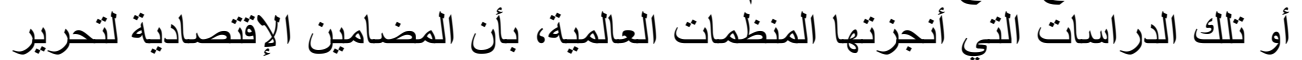

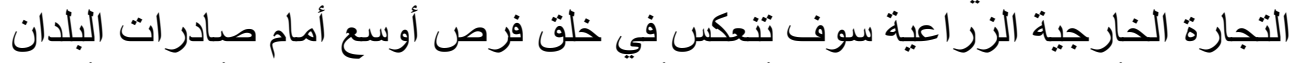

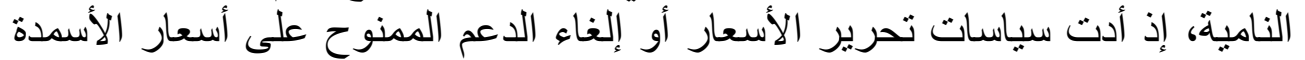

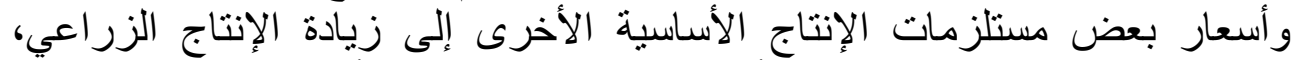

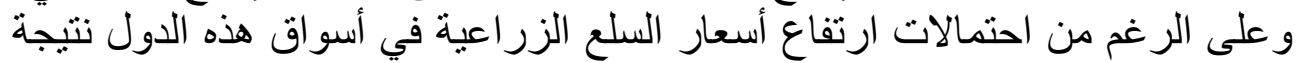

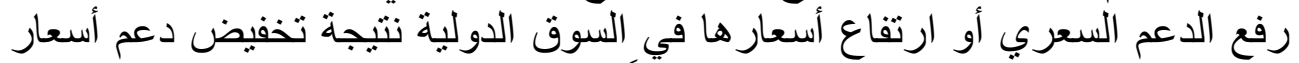

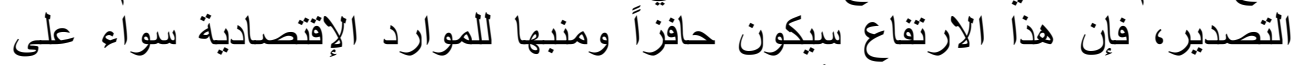

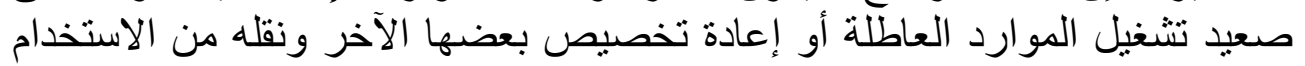

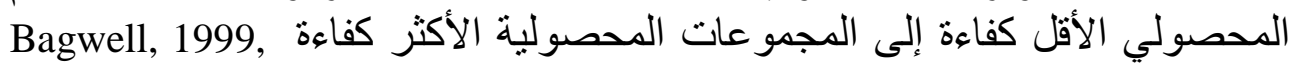
.(Staiger, 215-248)

أظهر التحليل أيضاً أهمية نسبة العمالة الزراعية إلى إجمالي العمل، ولكن

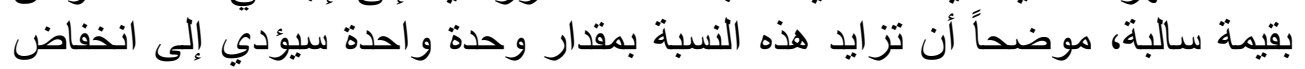

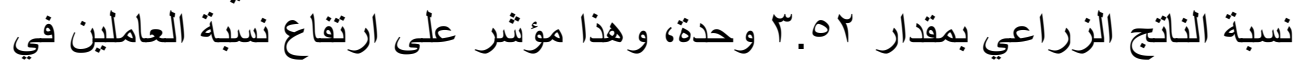

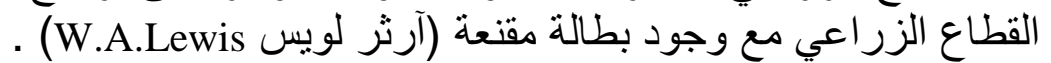

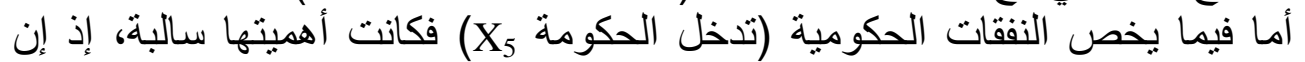

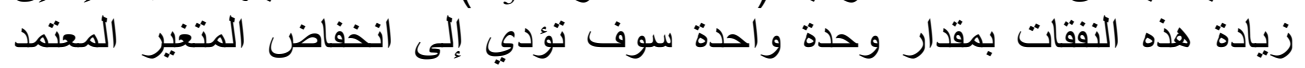

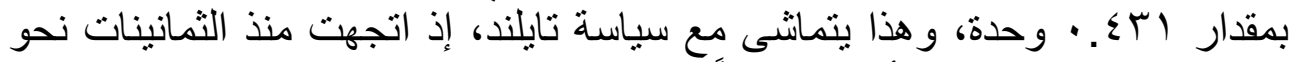

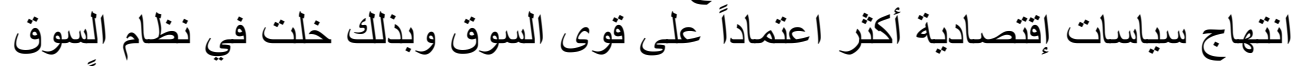

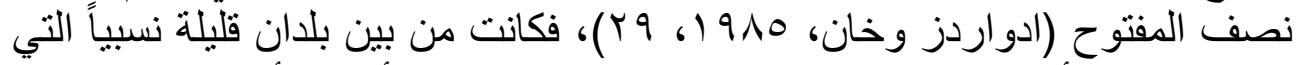

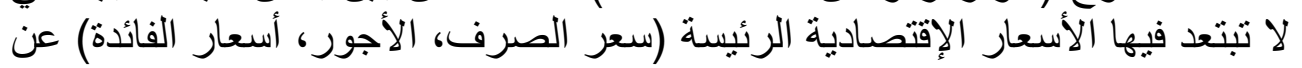

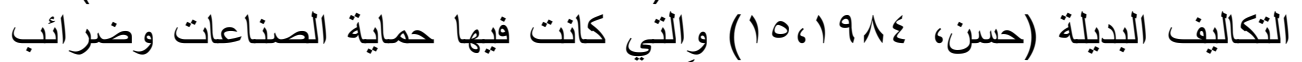

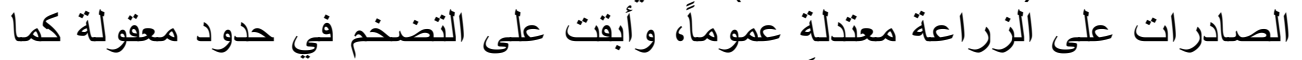
أبقت على سعر الصرف تنافسياً، مما أسهم إلى حدٍ كبير في نجاحها التها التصديري، كما 


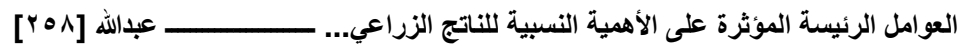

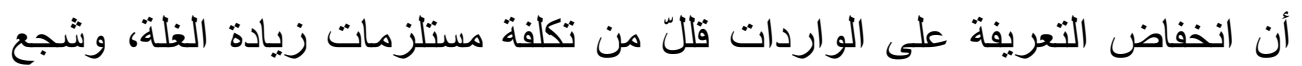

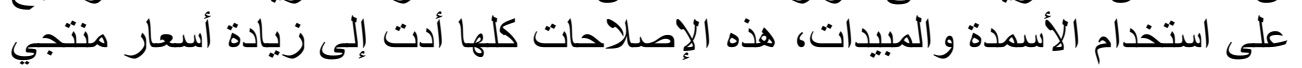

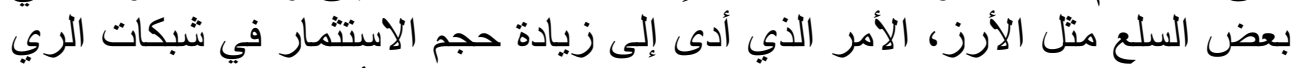

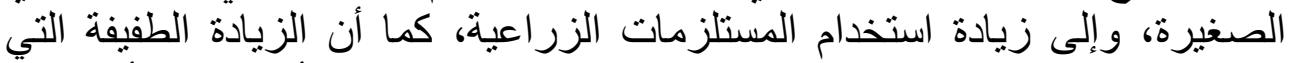

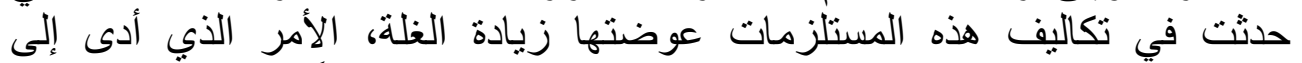
انخفاض التكاليف المتغيرة لوحدة الإنتاج فانعكس ذلك إيجابياً على زيّادة الإنتاج إلى لإنى وللت:عرف على أهمية كل متغير من المتغيرات المستقلة في نسبة الناتج

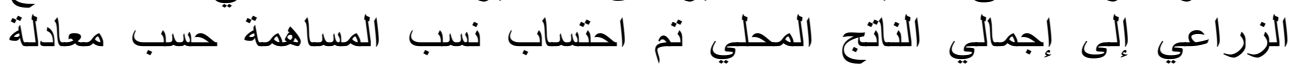
$\mathrm{Y}=0.167$ (14.17/6.26)(8.22) - 0.334 (14.17/59.31) (22.3) -

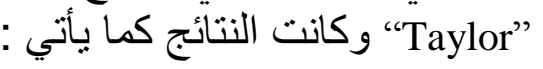
$0.364(14.17 / 616)$

$$
(1024)+0.048(14.17 / 98.70)(202)-0.083(14.17 / 11.56)(4.01)-
$$

$0.437(14.17 / 2260)(802)$

$$
\text { (x6) }
$$

وبذلك تحتل التقانة البايولوجية (X3) المرتبة الأولى في مساهمتها في نسبة

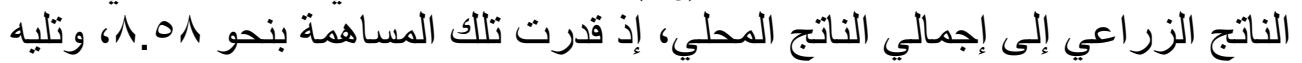

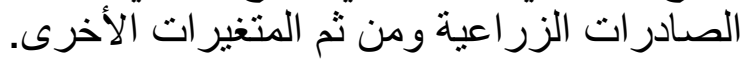

ثانياً- تركيا

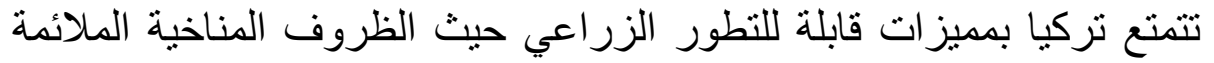

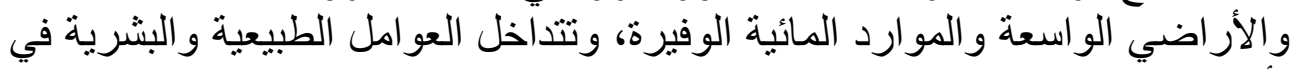

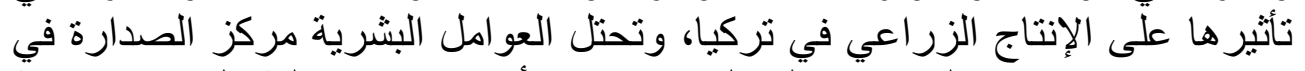

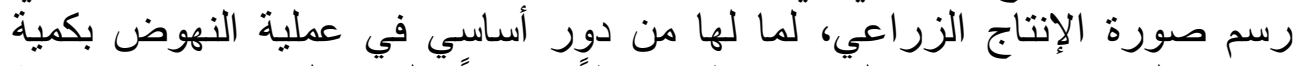

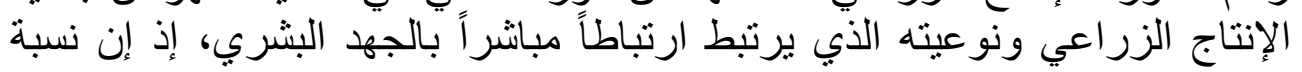

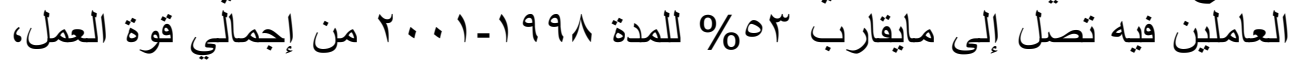

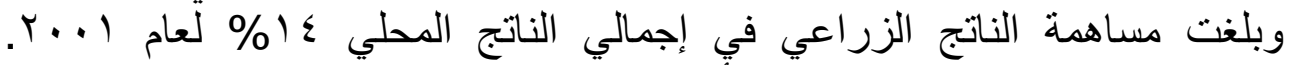

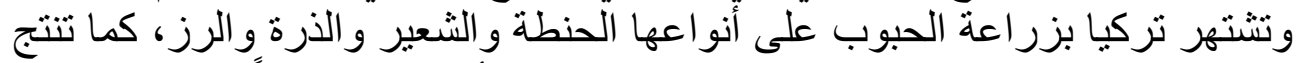

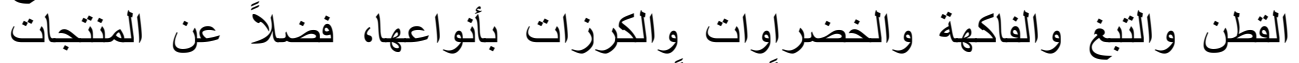

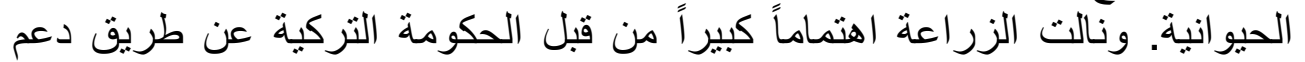

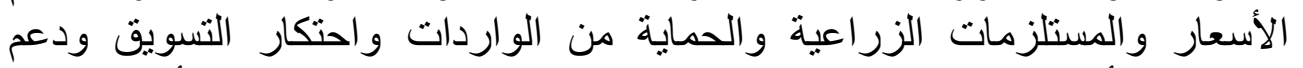

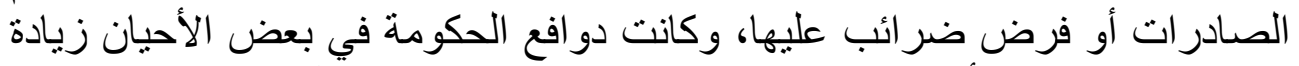

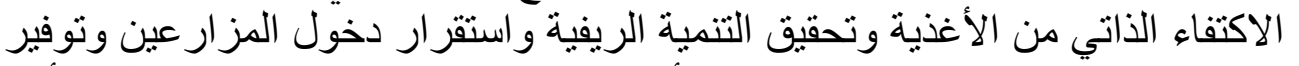

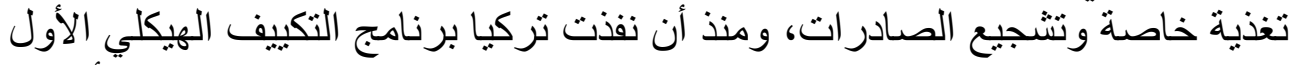

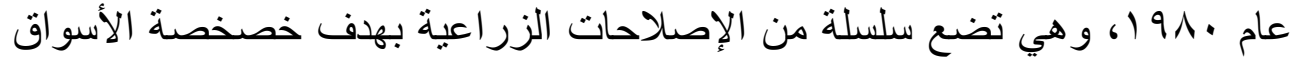


وتخفيض دعم الزراعة، وإلغاء الحواجز التجارية واندماج تركيا في الإقتصاد

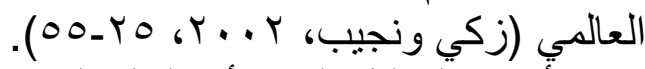
أُظهر ألتحليل الكمي أن الدالة الخطية هي أفضل الدوال الديل الدقدرة وتكون بالثكل

$$
\begin{aligned}
& \mathrm{Y}=18.4+0.864 \mathrm{X}_{1}-0.291 \mathrm{X}_{5} \\
& \mathrm{t}^{*}=(15.38)(12.58) \quad(-3.01) \\
& \mathrm{R}^{-2}=91.5 \% \\
& \mathrm{~F}=86.3 \quad \text { D.W }=1.88
\end{aligned}
$$

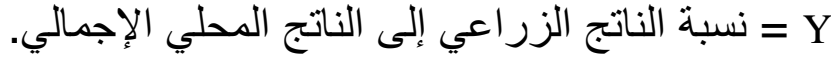

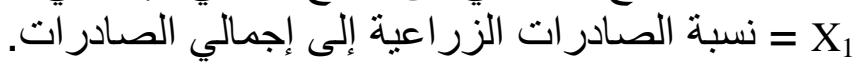

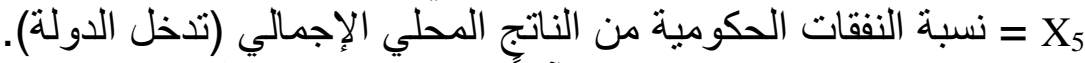

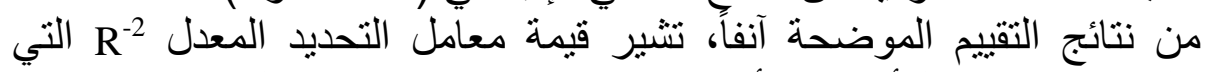

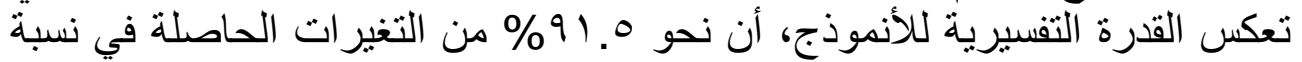

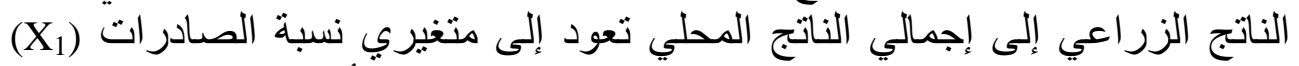

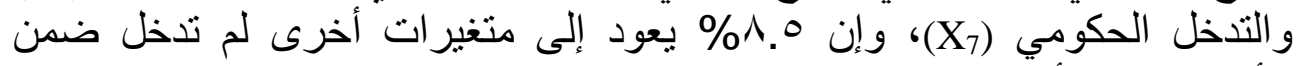

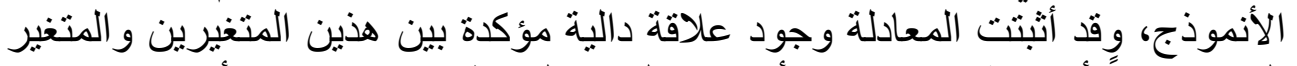

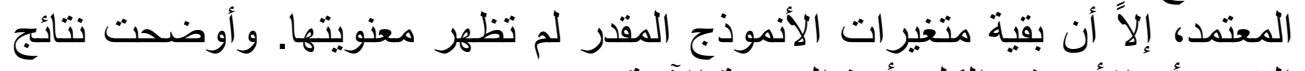
التقدير أن الأنموذج الكلي أخذ الصيغة الآتية:

$$
\begin{aligned}
\mathrm{Y}= & 19.9+0.944 \mathrm{X}_{1}-0.141 \mathrm{X}_{2}+0.003 \mathrm{X}_{3}+0.002 \mathrm{X}_{4} \\
\mathrm{t}^{*}= & (3.62) \quad(5.61) \quad(-1.56) \quad(0.98) \quad(0.53) \\
& -0.515 \mathrm{X}_{5}+0.001 \mathrm{X}_{6} \\
& (-2.72) \quad(1.01) \\
\mathrm{R}^{-2}= & 92.0 \quad \mathrm{D}) \mathrm{W}=2.22 \\
\mathrm{~F}= & 41.14 \quad
\end{aligned}
$$

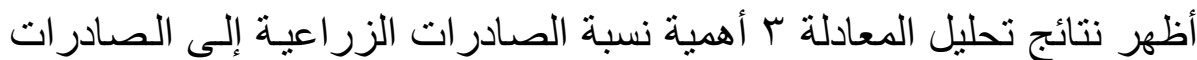

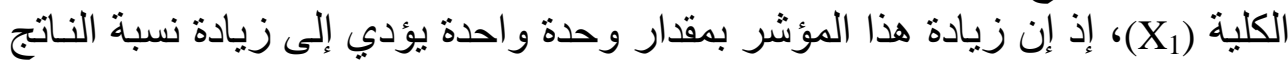

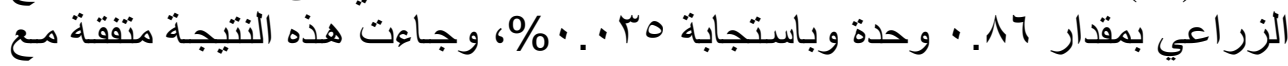

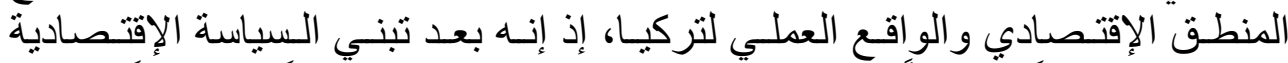

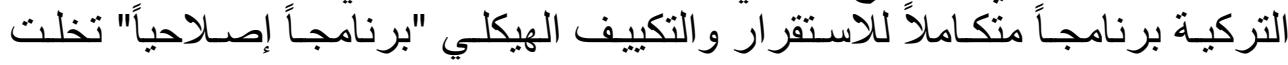

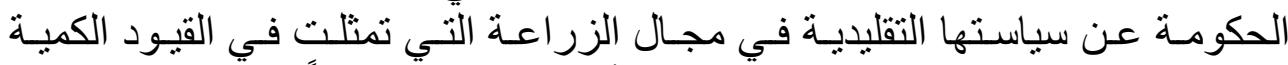

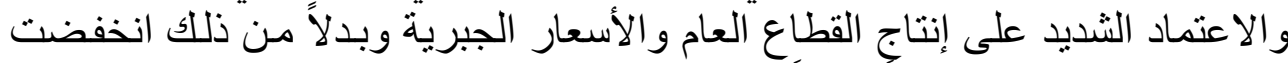

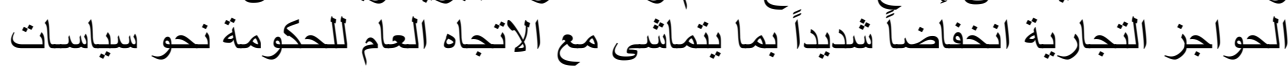

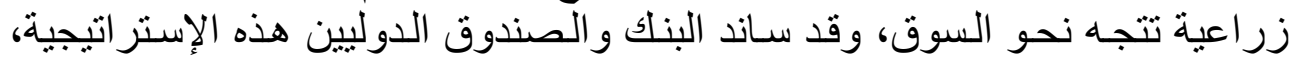

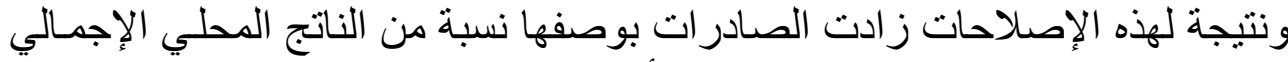
من حيث القيمة والحجم على السو اء، مما أدى إلى زيادة الاستثمار ات الزئل الزراعية بشكل 


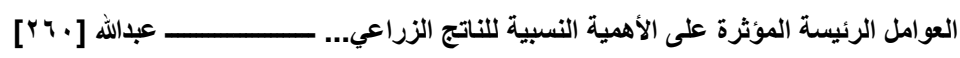

كبير، ومن ثم تأثير ها في زيادة الناتج الزر اعي. من جانب آخر تبين أن هناك تأثنير اً

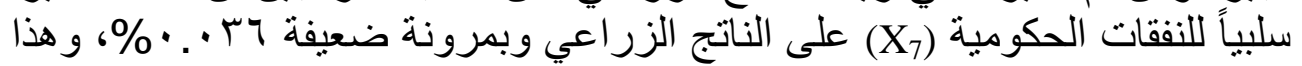

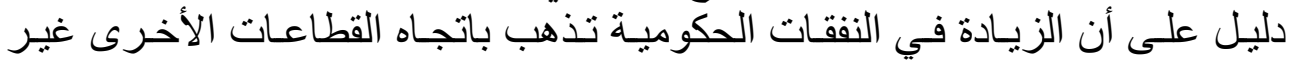

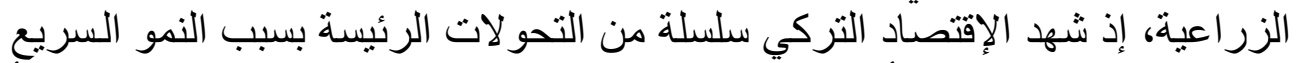

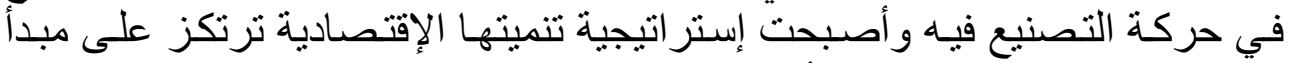

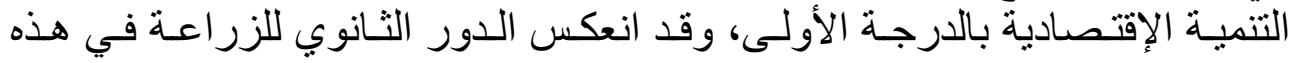

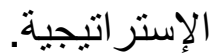
وبإحتساب نسبة مساهمة كل متغير من المتغيرات المستقلة توصلنا إلى النتيجة الآتية $\mathrm{Y}=0.195(18.38 / 3.69)(12.47)-0.420(18.38 / 44.40)(8)-0.009$

$(18.38 / 713.3)(404)+0.09(18.38 / 2.82)(0.31)-0.072(18.38 / 10.94)$

$(6.86)+0.197(18.38 / 1972.4)(512)$

يلاحظ أن نسبة الصادرات الزراعية إلى إجمالي الصادرات (X)

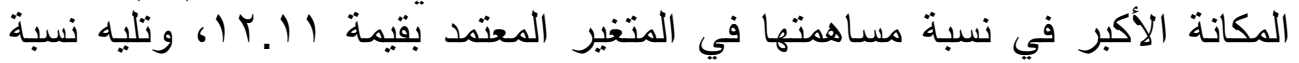
العمالة الزر اعية من إجمالي العمالة.

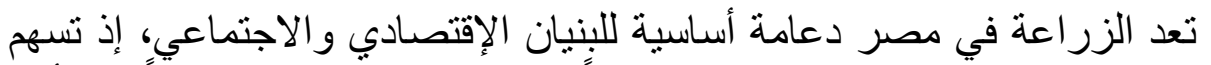

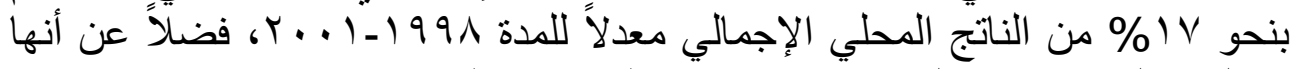

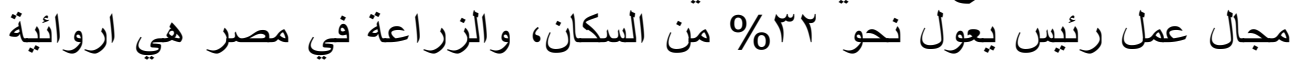
مؤكدة بنسبة 90\%، مستندة إلى الطاقة التخزينية الكبيرة لثبكة الري الري التي كونت

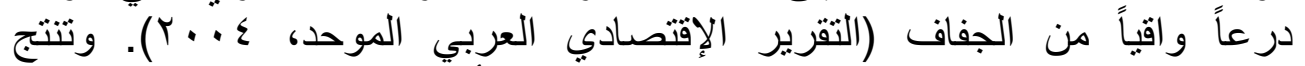

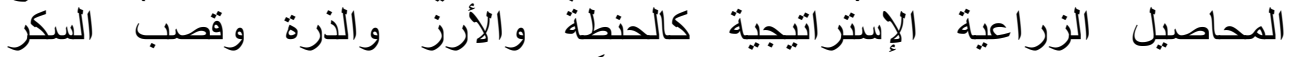

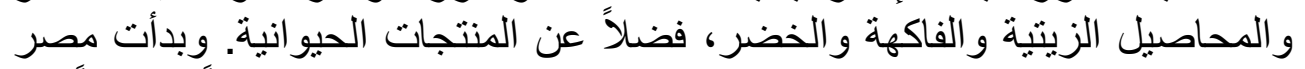

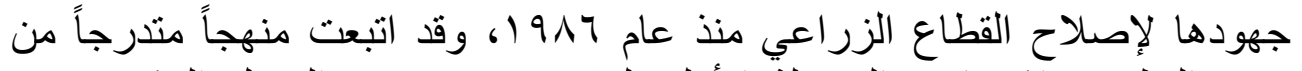

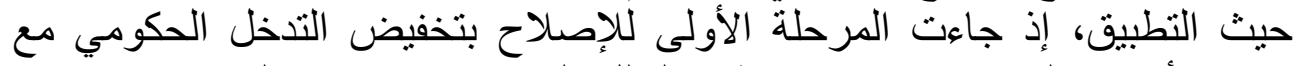

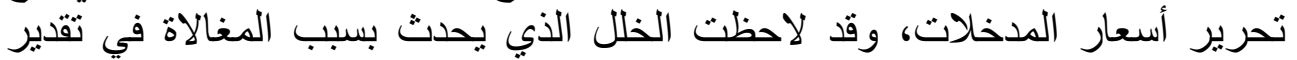

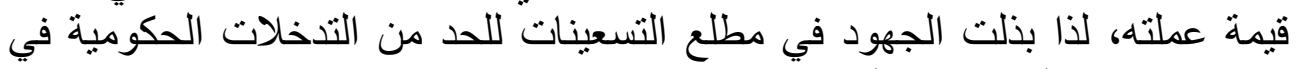

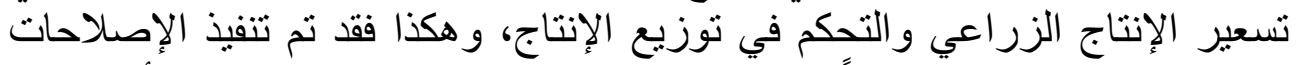

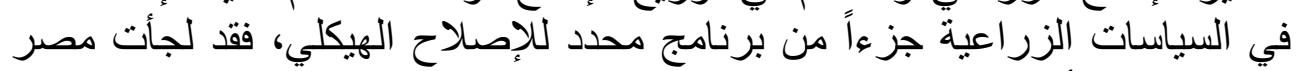

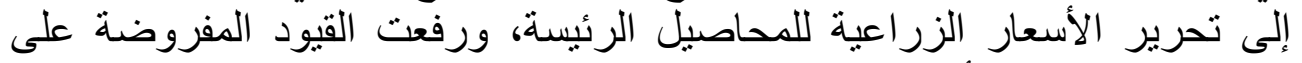

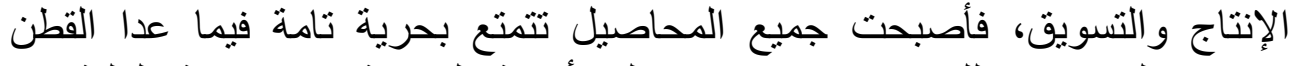

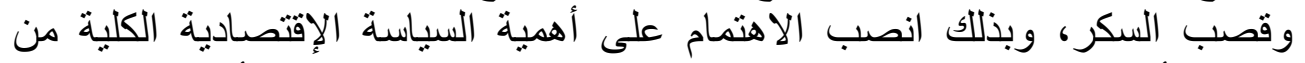

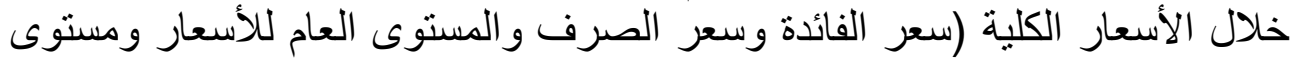


الأجور)، لاستعادة التوازن بين الطلب الكلي و العرض الكلي للموارد في الإقتصاد

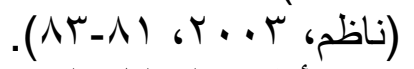
أظهر التحليل الكمي أن الدالة الخطية هي أفضل الدوال المقدرة وهي بالثكل $\mathrm{Y}=5.27+0.143 \mathrm{X}_{2}+1.07 \mathrm{X}_{3}+0.282 \mathrm{X}_{5}$

$\mathrm{t}^{*}=(3.25) \quad(3.63)$ الآتي: - n

$\mathrm{R}^{-2}=87.6 \%$

$\mathrm{F}=50.36$

D.W $=1.85$

: إذ إن

نسبة الناتج الزر اعي إلى الناتج المحلي الإجمالي = Y

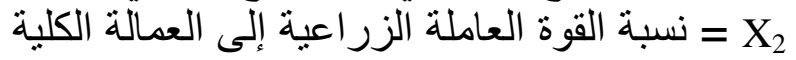
= X3

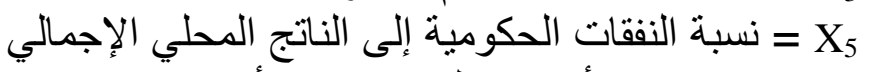

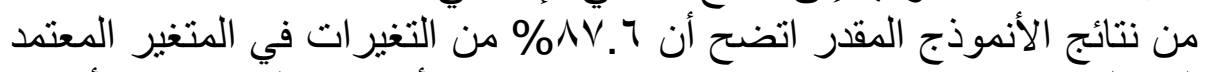
تعود إلى كل من (X) فلم تظهر معنوياتها. وأوضحت نتائج التقدير أن الأنموذج الكلي أخذ الصيغة الآتية: $\mathrm{Y}=12.2+0.696 \mathrm{X}_{1}+0.055 \mathrm{X}_{2}+1.35 \mathrm{X}_{3}-0.007 \mathrm{X}_{4}+0.115 \mathrm{X}_{5}-0.48 \mathrm{X}_{6}$ $\mathrm{t}^{*}=(3.23)$ $\mathrm{R}^{-2}=88.4 \%$ $\mathrm{F}=27.72 \quad$ D. $\mathrm{W}=2.13$

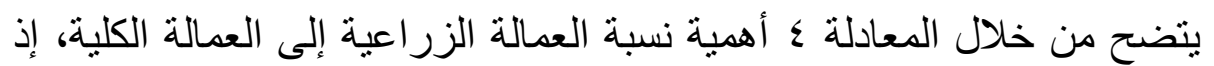

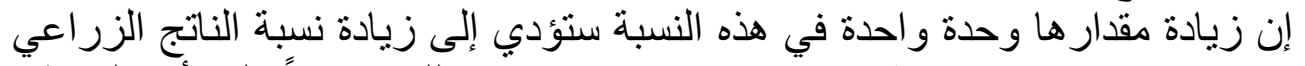

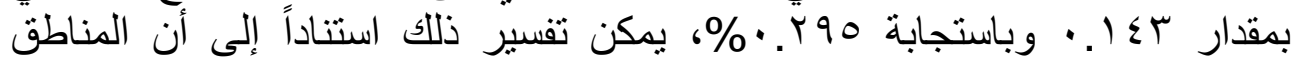

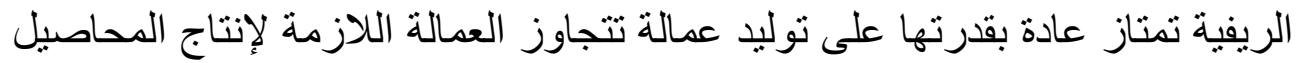

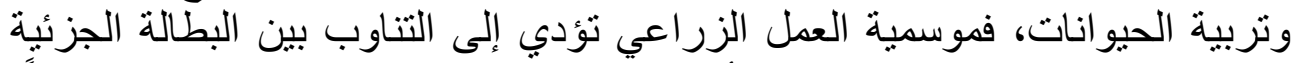

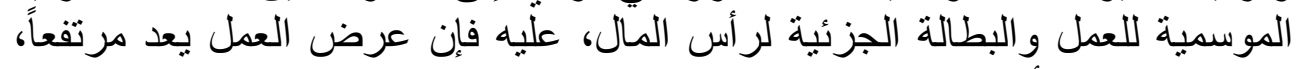
ومن ثم تتجه الأجور نحو الانخفاض فتنخفض بذألك لكالك تكاليف الإنتاج ويزداد الناتج

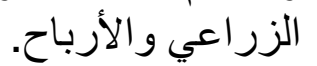

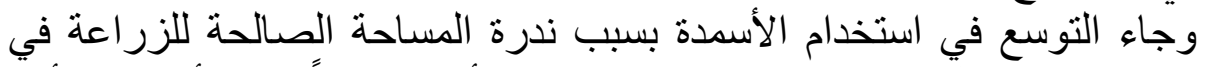

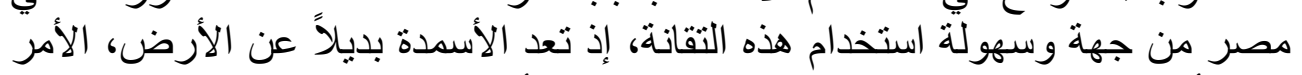

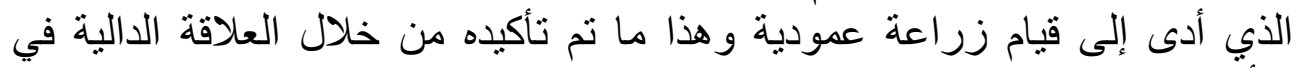

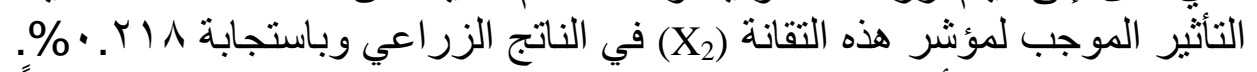

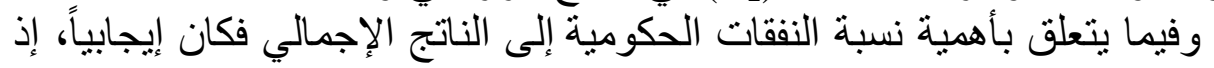

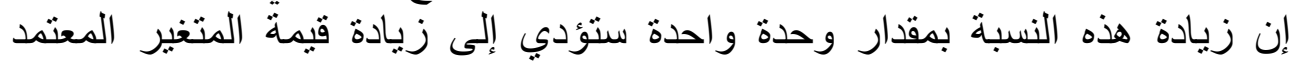

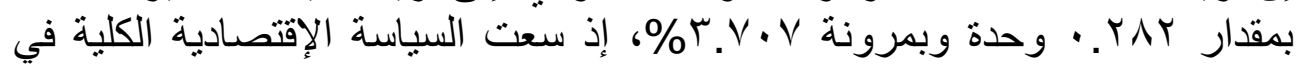

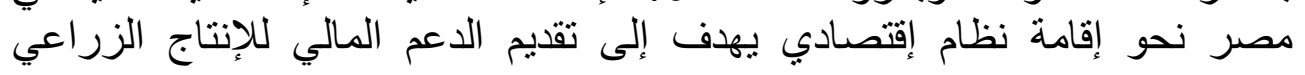




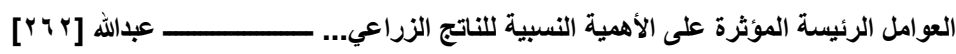

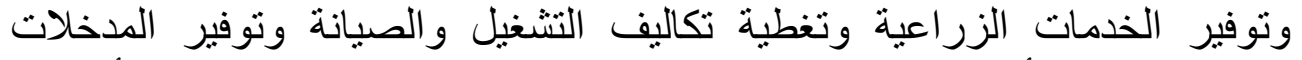

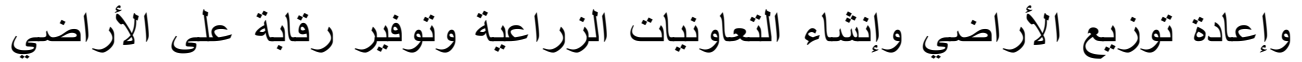

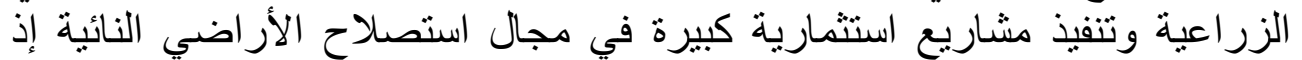

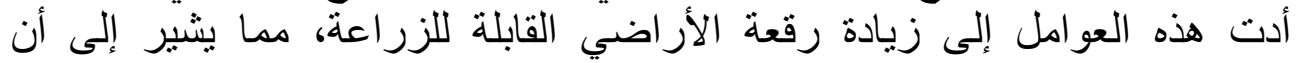

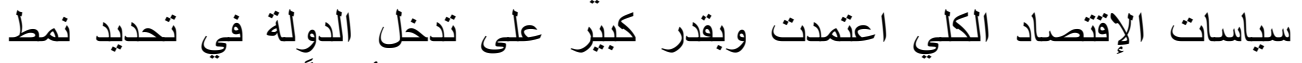

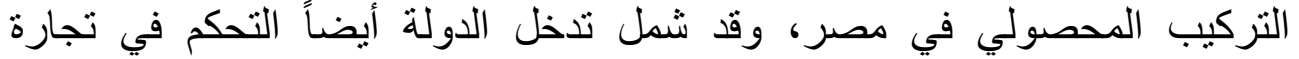
المنتجات الزراعية النقدية التي تعد المنتجات الرئيسة التي يعتمد عليها التيها الإقتصاد

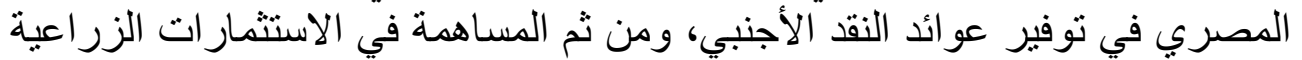

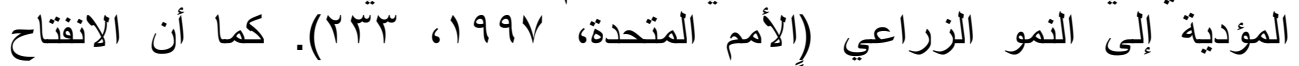
الإقتصادي قد نرتب عليه جزئياً ابتعاد مصر عن زراعات الاعت تتسم بميزة نسبية عالية

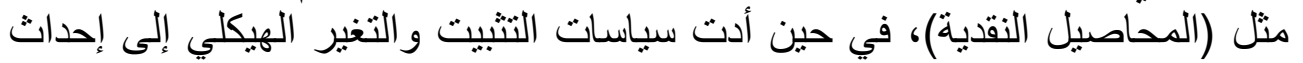

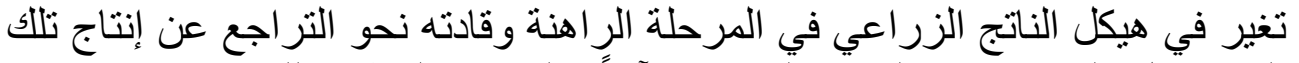

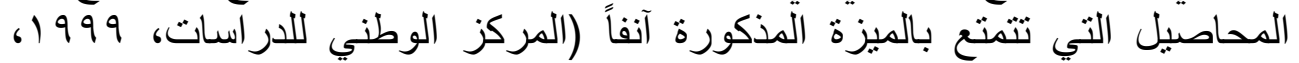
وللتعرف على أهمية كل متغير من المتغيرات المؤثرة في نسبة الناتج

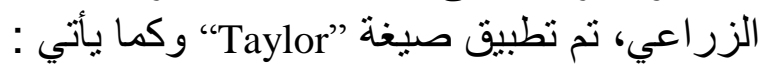
$\mathrm{Y}=0.024(18.40 / 9.09)(2)+0.124(18.40 / 36.77)(12)+0.267(18.40 / 3.90)(1.593)$

$$
-0.018(18.40 / 76.31)(4)+0.271(18.40 / 13.09)(9)-0.180(18.40 / 5.55)(3.186)
$$

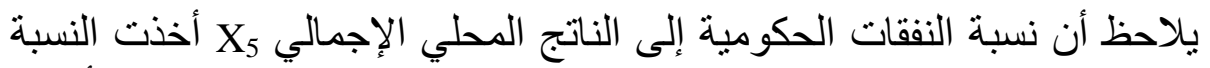

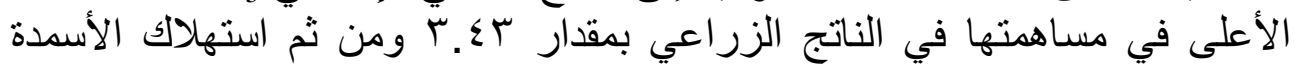

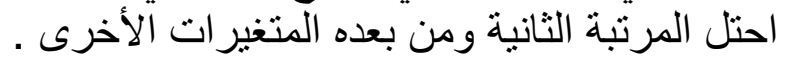

تعد بنغلادش دولة زراعية، إذ تشكل الأراضي الصالحة للزراعة إعة فيها نسبة رابعاً- بنغلادش

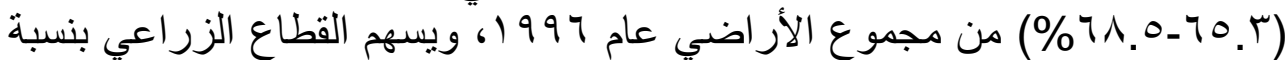

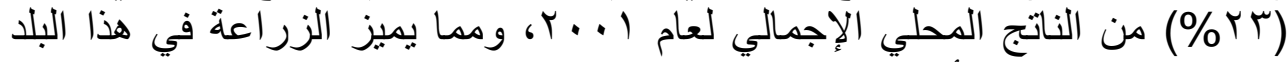

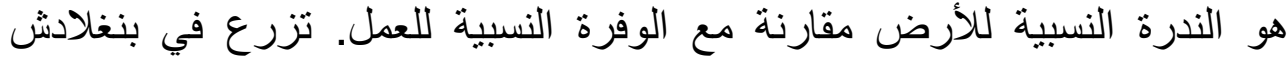

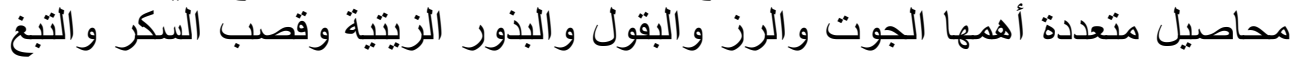

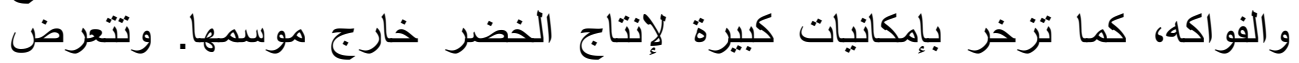

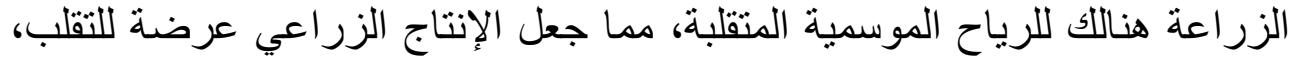

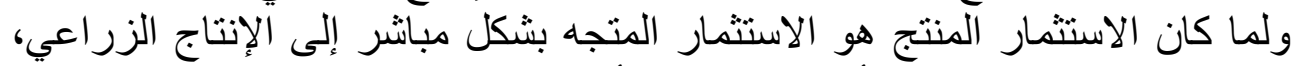

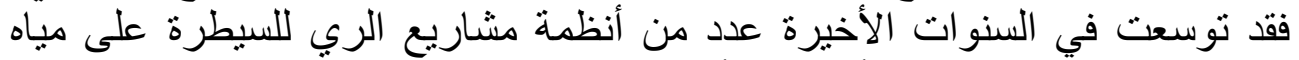

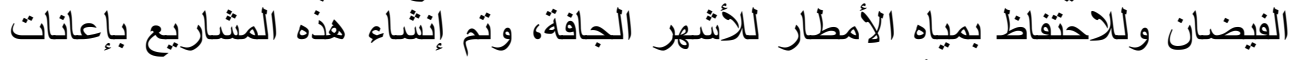
واسعة من الدولة، كما أنها تعمل تحت إثراف الف الدولة مع فرض رسوم سنوية ثابتة 
على الفلاحين بوصفها خدمات ري، وتعكس هذه الرسوم درجة الإعانات التقيلة التي

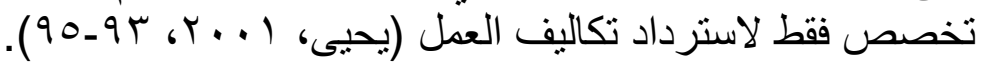

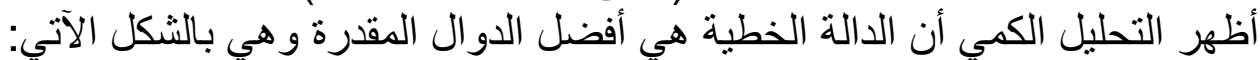
$\mathrm{Y}=69.4-0.004 \mathrm{X}_{1}-1.0 \mathrm{X}_{2}-1.26 \mathrm{X}_{5}$
$\mathrm{t}^{*}=(22.73)(-2.05)$
$(-12.92) \quad(-3.20)$
المعادلة ه

$\mathrm{R}^{-2}=90.2$

$\mathrm{F}=65.12$

D.W $=1.86$

إذ إن :

= نسبة الناتج الزر اعي إلى الناتج المحلي الإجمالي = Y

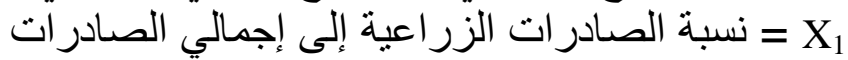

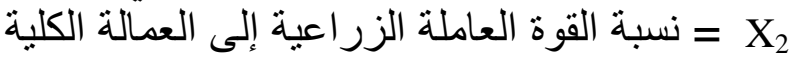

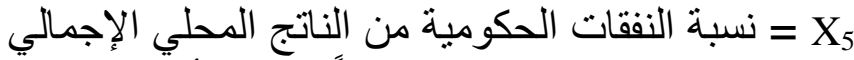

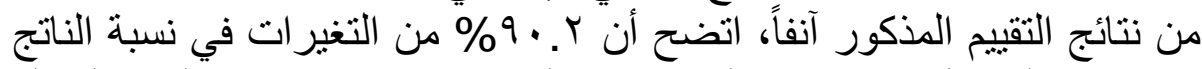

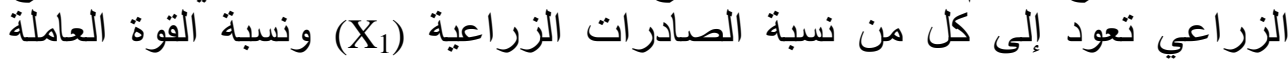

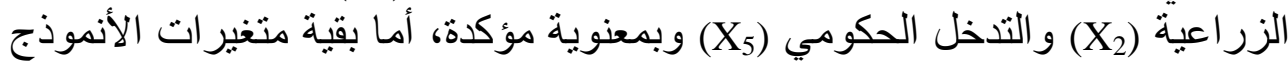

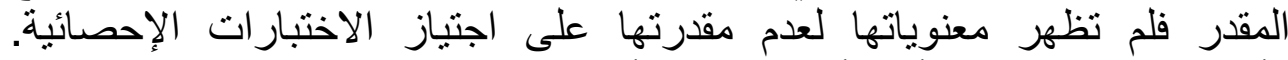
و أوضحت نتائج التقدير أن الأنموذج الكلي أخذ الصيغة الآنية : $\mathrm{Y}=92.7-0.005 \mathrm{X}_{1}-1.84 \mathrm{X}_{2}+4.42 \mathrm{X}_{3}-0.132 \mathrm{X}_{4}-1.54 \mathrm{X}_{5}+1.29 \mathrm{X}_{6}$ $\mathrm{t}^{*}=\left(\begin{array}{lllll}4.10) & (-2.02) & (-2.35) & (0.91) & (-0.35)\end{array}\right.$ $\mathrm{R}^{-2}=89.3$ $\mathrm{F}=30.35 \quad$ D.W $=2.04$ من المعادلة ه تبين أهمية أن تأثثر نسبة الصادرات الزراعية إلى إجمالي

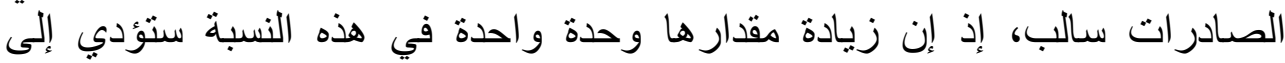

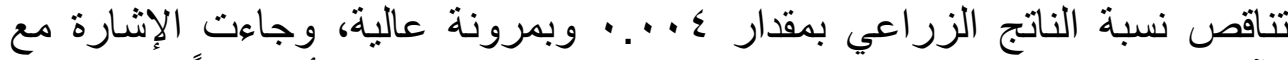

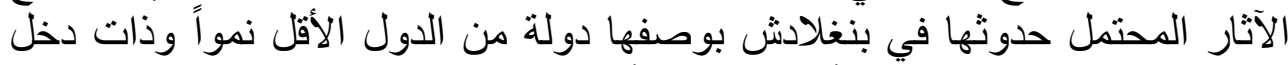

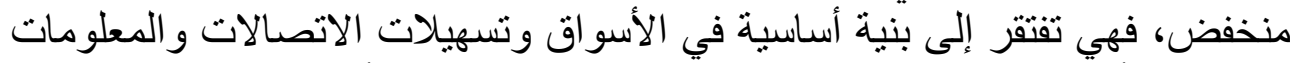

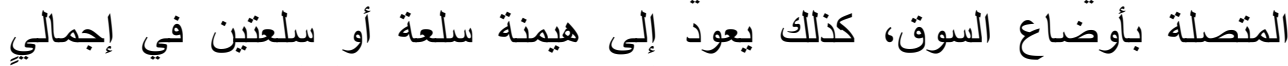

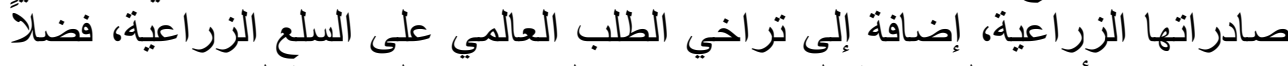

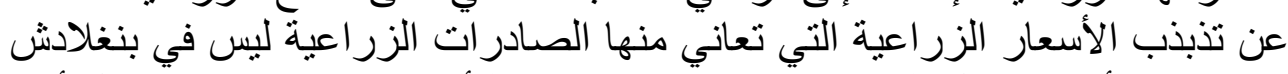

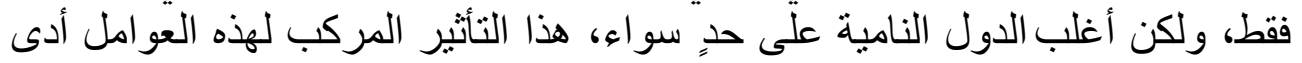
إلى خفض في مستوى المحفز ات المقدمة لتشجيع إنتاج محاصيل التهاءئ التصدير.

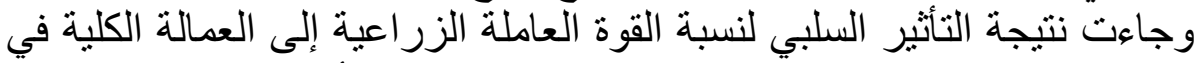

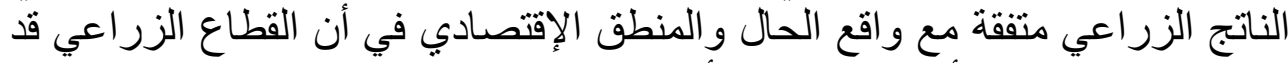

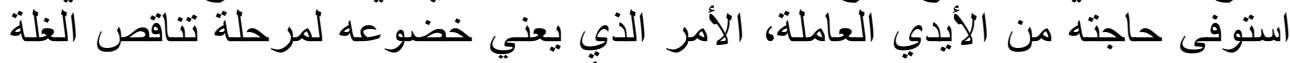

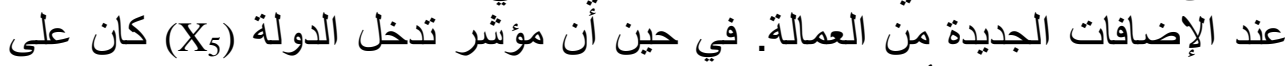

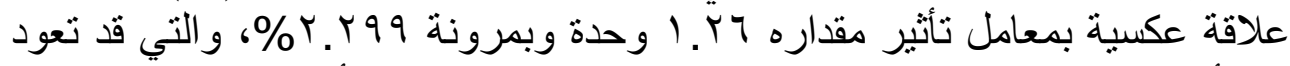

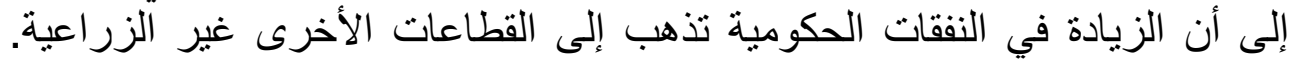




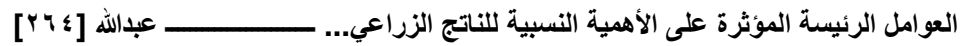

وبالاعتماد على معادلة "Taylor" تم احتساب نسب مساهمة المتغير ات المستقلة في

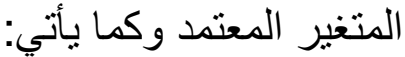
$Y=-0.180(28.09 / 185.71)(326.13)-2.20(28.09 / 34.81(8.44)+0.148$

$$
\text { (x1) (x2) }
$$

$(28.09 / 1.07)(1.236)-0.0175(28.09 / 4.96)(3.56)-0.216(28.09 / 4.5)(2)$

$+0.124(28.09 / 2.50)(1.54)$

(x4)

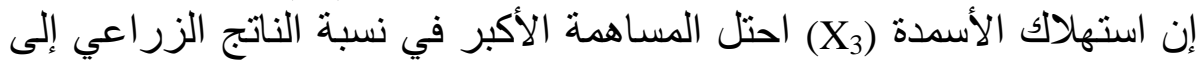

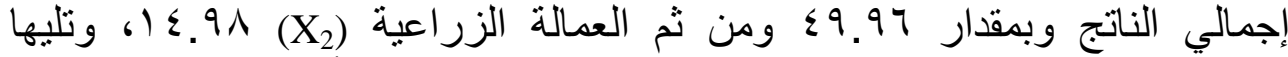

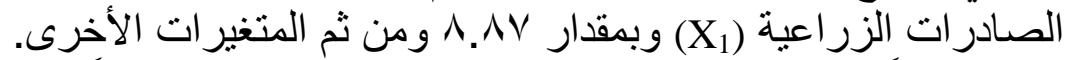

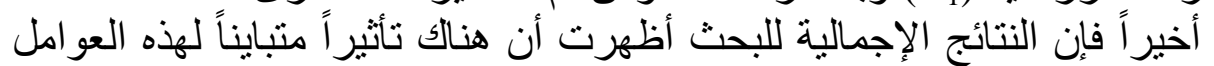

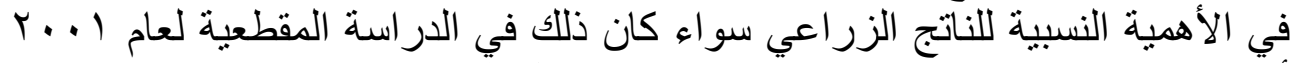

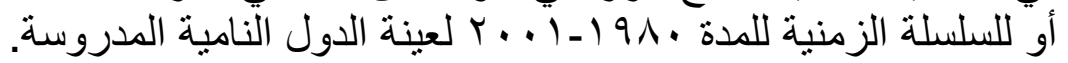

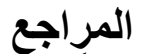 \\ أولاًا المراجع باجع باللغة العربية}

ا. الأمم المتحدة، حالة الأغذية والزراعة، الاعة الصناعات الزراعية والتنمية الإقتصادية، منظمة

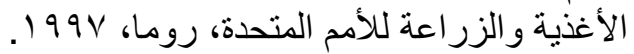

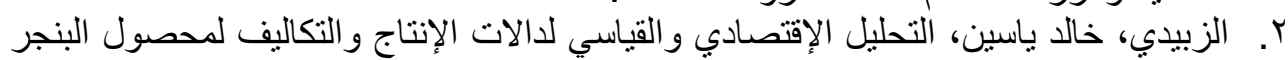

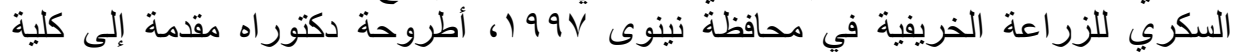

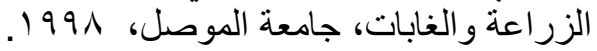

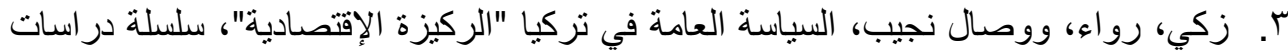

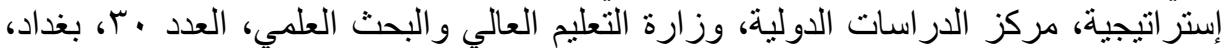
r... r ع. السيفو، وليد إسماعيل، المدخل إلى الإقتصاد القياسي، وزارة التعليم العالي والبحث العلمي،

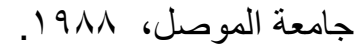
๑. عبد الفضيل، محمود، العرب والتجربة الأسيوية "الدروس المستفادة"، مركز دراسات الوحدة

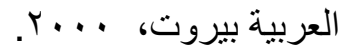

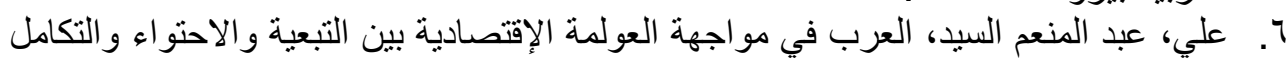

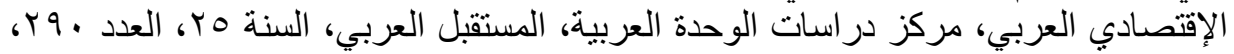

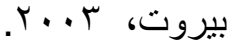

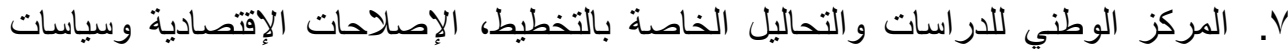
الخوصصة في البلدان العربية، بحوث الندوات الندوة الفكرية، مركز دراسات الوحدة العربية،

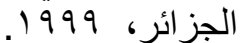
^. ناظم، قيس، اثر العولمة في الأداء الزراعي في بلدان نامية مختارة، أطروحة دكتور اه مقدمة

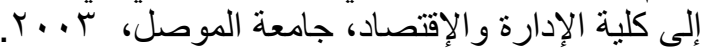

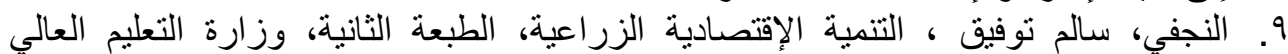

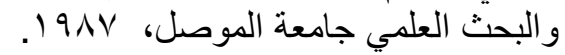
• 1. النجفي، سالم توفيق، التجارة الخارجية التوجية الزراعية "مبادئ ومتضمنات"، وزارة التعليم العالي

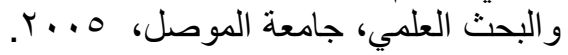


$(r \cdot)^{9} \cdot$

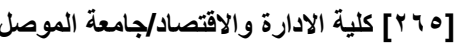

1 ا. النجفي، سالم توفيق، الزر اعة العربية و إقتصاد العولمة "مقاربات إقتصادية"، تنمية الرافدين،

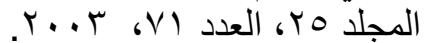

r ا. النجفي، سالم توفيق، موروثات القرن العشرئ العشين "مقاربات إقتصادية"، بيت الحكمة، بغداد، r...

با.يحيى، إنعام، تأثير التغيرات الهيكلية على الأمن الغذائي في دول نامية مختارة، أطروحة

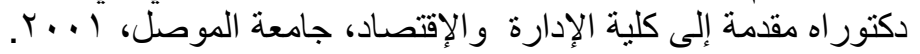

1. G. S. Maddala, Econometrics, McGraw-Hill, International book Company, Singapore, 1977.

2. Human Development Report, United Nations Development Programmer, Oxford, Oxford university Press, New York, 2003.

3. Kyle Bagwell and Robert W, Staiger, An Economic Theory of Gatt, The American Economic Review, Vol , 89, No, 1,1999.

4. M. Ram, Trade: An Engine of Economic Development in Asian Countries Rivista Int, Sci, Economic Com, Vol, 30, No, 9,1983.

5. R. S. Pindyck and Rubifeld D, L, Econometric Model and Economic Forecasts, McGraw-Hill, Kogakusha Ltd, Tokyo, 1976.

6. Ray, A, Prices and Trade Polices in the International Agriculture, Finance and Development, International Monetary Fund, Washington, Vol, 23, No. 3,1986.

7. S. Gupta, Export and Economic Growth, The Indian Economic Journal, Vol.32 ,No. 3,1984 .

8. The world Bank, World Development Indicators, Washington, USA, 2003.

9. The World Bank Group, Data and Statistics, 2002.

10. http://www, worldbank, data/countryclass

11. W. Tyler, Growth and Export Expansion in Developing Countries "Some Empirical Evidence", Journal of Development Economics, Vol. 9,1981. 